\title{
Shock Waves as a Treatment Modality for Spasticity Reduction and Recovery Improvement in Post-Stroke Adults - Current Evidence and Qualitative Systematic Review
}

This article was published in the following Dove Press journal:

Clinical Interventions in Aging

\section{Robert Dymarek (D) Kuba Ptaszkowski iD ${ }^{2}$ Lucyna Ptaszkowska (D) ${ }^{3}$ Mateusz Kowal id ${ }^{3}$ Mirosław Sopel (D) Jakub Taradaj (iD) ${ }^{4,5}$ Joanna Rosińczuk (D) \\ 'Department of Nervous System Diseases, Wroclaw Medical University, Wroclaw, Poland; ${ }^{2}$ Department of Physiotherapy, Wroclaw Medical University, Wroclaw, Poland; \\ ${ }^{3}$ Department of Physiotherapy, Opole Medical School, Opole, Poland; ${ }^{4}$ Institute of Physiotherapy and Health Sciences, Academy of Physical Education, Katowice, Poland; ${ }^{5}$ College of Rehabilitation Sciences, University of Manitoba, Winnipeg, Canada}

Purpose: This systematic review examines intervention studies using extracorporeal shock wave therapy (ESWT) application in post-stroke muscle spasticity with particular emphasis on the comparison of two different types of radial (rESWT) and focused shock waves (fESWT).

Methods: PubMed, PEDro, Scopus, and EBSCOhost databases were systematically searched. Studies published between the years 2000 and 2019 in the impact factor journals and available in the English full-text version were eligible for inclusion. All qualified articles were classified in terms of their scientific reliability and methodological quality using the PEDro criteria. The PRISMA guidelines were followed and the registration on the PROSPERO database was done.

Results: A total of 17 articles were reviewed of a total sample of 303 patients (age: 57.87 \pm 10.45 years and duration of stroke: $40.49 \pm 25.63$ months) who were treated with ESWT. Recent data confirm both a subjective (spasticity, pain, and functioning) and objective (range of motion, postural control, muscular endurance, muscle tone, and muscle elasticity) improvements for post-stroke spasticity. The mean difference showing clinical improvement was: $\Delta=34.45 \%$ of grade for fESWT and $\Delta=34.97 \%$ for rESWT that gives a slightly better effect of rESWT $(\Delta=0.52 \%)$ for spasticity $(\mathrm{p}<0.05)$, and $\Delta=38.83 \%$ of angular degrees for fESWT and $\Delta=32.26 \%$ for rESWT that determines the more beneficial effect of fESWT $(\Delta=6.57 \%)$ for range of motion $(\mathrm{p}<0.05)$, and $\Delta=18.32 \%$ for fESWT and $\Delta=22.27 \%$ for rESWT that gives a slightly better effect of $\operatorname{rESWT}(\Delta=3.95 \%)$ for alpha motor neuron excitability $(\mathrm{p}<0.05)$. The mean PEDro score was $4.70 \pm 2.5$ points for fESWT and $5.71 \pm 2.21$ points for rESWT, thus an overall quality of evidence grade of moderate ("fair" for fESWT and "good" for rESWT). Three studies in fESWT and four in rESWT obtained Sackett's grading system's highest Level 1 of evidence.

Conclusion: The studies affirm the effectiveness of ESWT in reducing muscle spasticity and improving motor recovery after stroke.

Keywords: shock waves, neurorehabilitation, stroke, muscle spasticity, older adults, systematic review

\section{Introduction}

\section{Post-Stroke Muscle Spasticity}

Spasticity is a common phenomenon and the primary motor impairment after stroke, affecting patients' motor recovery and challenging both researchers and clinicians. ${ }^{1}$ 
Global data estimated that over 12 million people suffer from muscle spasticity localized in the extremities. ${ }^{2}$ Studies have shown that $19 \%$ of the post-stroke subjects demonstrate spasticity within 3 months of their stroke, reaching to even $40-70 \%$ in chronic phase of stroke. ${ }^{3}$

The most cited Lance definition of muscle spasticity describes it as 'a motor disorder characterized by a velocitydependent increase in tonic stretch reflexes (muscle tone) with exaggerated tendon jerks, resulting from hyperexcitability of the stretch reflex, as one component of the upper motor neuron (UMN) syndrome. ${ }^{4}$ However, there is still no agreement on the definition of spasticity, which is an indication of the complex nature and diversity of these phenomena. ${ }^{5}$ Trompetto et $\mathrm{al}^{6}$ pointed out that in patients with UMN syndrome, weakness causes strongly decreased mobilization of the affected muscles. Immobilization in a shortened position leads to muscle contracture, which is the cause of a specificity. At the same time, muscle immobilization reduces post-activation depression, which is a key mechanism for the development of spasticity.

\section{Consequences of Muscle Spasticity}

Clinically, the spasticity of muscles is related to increased muscle tension, stiffness, excessive reflexes, and possible joint spasms. ${ }^{7}$ In combination with reduced voluntary muscle strength, postural imbalances, and weakened motor control, this leads to significant functional limitations. The pathophysiology underlying the histological changes in the skeletal muscle that contribute to these functional deficits is not clearly understandable. ${ }^{8}$ It is also well known that the emergence and development of spasticity can be affected by the structural changes in fibers of muscle and tendon as well as mechanical or morphological changes of intra- and extracellular components. ${ }^{8,9}$ In addition, muscle spasms occur in addition to spasticity due to a reduction in the length of muscle fibers and a decrease in the number of sarcomeres in the muscle fibers. ${ }^{10}$

Most patients suffering from spasticity are unable to work effectively and are precluded from job activity. ${ }^{11}$ It has been shown in health economic studies that the direct costs are four times bigger for patients with spastic paresis than for those without spasticity. ${ }^{12}$ For this reason, there is still a need to find more effective and beneficial methods of neurorehabilitation for spasticity management.

\section{Management of Post-Stroke Spasticity}

There is a wide range of treatment procedures for the management of spasticity that includes anti-spastic drugs, intrathecal baclofen, alcohol and botulinum toxin injections, surgical interventions, and rehabilitation. ${ }^{13,14}$ Also, biophysical agents, such as neuromuscular stimulation (NMS), electromyography neuromuscular stimulation (EMG-NMS), transcutaneous electrical nerve stimulation (TENS), functional electrical stimulation (FES), or even ultrasound therapy are extensively used as adjunctive treatments. ${ }^{15-17}$ Unfortunately, there is still limited evidence that procedures like transcranial magnetic stimulation (TMS) or transcranial direct current stimulation (tDCS) reduce spasticity in cases of advanced, non-invasive neurostimulation and neuromodulation techniques. One of today's promising methods, though still not commonly used by clinicians, is extracorporeal shock wave therapy (ESWT), also known as shock waves.

\section{Shock Wave Technology and Therapy}

From a biophysical point of view, shock waves are defined as a sequence of single, highly energetic, biphasic acoustic impulses characterized by rapid propagation of suddenly increased pressure in three-dimensional space applied directly into the tissues without affecting their global destruction. There are two types of ESWT generators, focused ESWT (fESWT) and unfocused-radial ESWT (rESWT). ${ }^{18}$ The fESWT is typically generated by electromagnetic, electrohydraulic, and piezoelectric sources. In this method, the pressure increases rapidly from under 10 ns up to $100-1000$ bars (energy absorbency to the depth of about $12 \mathrm{~cm}$ ). On the other hand, in the rESWT, the pressure increases slightly and much more slowly - up to $5 \mu$ s and reaching 1-10 bars (energy absorbency to the depth of $3 \mathrm{~cm}$ ). This is the reason fESWT is more intensive than rESWT within the focal area of the highest energy exposure when rESWT has the superficial region of interest. For this reason, the rESWT is deemed to be less invasive than FESWT thus it might be much more appropriate for physiotherapy purposes. ${ }^{19}$

One of the most significant ESWT parameter is the amount of energy administered during the single shock, i.e., energy flux density (EFD), which, for less-invasive procedures, ranges between 0.01 and $0.5 \mathrm{~mJ} / \mathrm{mm}^{2}$. During a single application, the number of 500-4000 impulses may be delivered with the optimal shots' frequency of strikes between 1 and $8 \mathrm{~Hz}^{20,21}$ The fESWT technology is the better-studied and has been successfully used since the late 1980s for physiotherapeutic purposes. In turn, the rESWT is a quite novel treatment, which has started to be used in 1999 thus the biological mechanisms following this therapy are still poorly investigated regarding a smaller amount of Level 1 and Level 2 studies. $^{22}$ 


\section{Neuronal Mechanisms of Shock Waves}

The mechanisms underlying clinical efficacy of ESWT in musculoskeletal pathologies are well documented. Based on the basic studies, it was revealed that ESWT promotes the activation of molecular and immunological reactions improving blood microcirculation, stimulating angiogenesis and increasing neovascularization, ${ }^{23,24}$ activation of the anti-inflammatory reaction, and suppression of leukocyte infiltration. ${ }^{25,26}$ Moreover, intensification of tissue regeneration, effective recruitment of fibroblasts, and decrease of the tissue apoptosis were observed. ${ }^{27}$

The mechanisms of ESWT action on spasticity due to central nervous system (CNS) injury are still unknown. Variable mechanisms have been proposed, such as the link between ESWT-related activation of nitric oxide (NO), which could modulate the neurocytes. ${ }^{28}$ Some animal experiments report regenerative properties of ESWT characterized by intense growth of axonal regeneration as a result of the partial destructive impact of ESWT. This phenomenon first removes the degenerated axons and then increases the ability to create new axons. ${ }^{29}$

Moreover, it was determined that low-energy ESWT enhances the neuroprotective effect of vascular endothelial growth factor (VEGF), ${ }^{30,31}$ as well as improving neurological function. ${ }^{32,33}$ Another experimental trial reported that early application of ESWT increased the expression of neurotrophin-3 (NTH-3), and day-to-day use of ESWT stimulated the activity of macrophages and Schwann cells, which contributes to the survival and regeneration of neurons. ${ }^{34}$ Further, ESWT stimulates neurogenesis by enhancing proliferation of neural stem cells (NSC). This may play an important role in the repair of brain function in CNS diseases. ${ }^{35}$

Experimental data report that Schwann cells treated with ESWT had significantly improved isolation, culture, and proliferative capacities, which is clinically important in the peripheral nervous system damage. ${ }^{36}$ Moreover, a postESWT reversible segmental demyelination of the largediameter fibers was shown to have a non-significant negative impact on their functioning. ${ }^{37}$ Moreover, it has been demonstrated that ESWT is successful in enhancing peripheral nerve function and has a beneficial effect on the reduction of denervation atrophy. ${ }^{38}$

\section{Current Evidence on Shock Waves}

According to the statements of the International Society for Medical Shockwave Treatment (ISMST), fESWT is a wellstudied physical modality and has been successfully used for physiotherapeutic purposes in a wide range of musculoskeletal conditions. ${ }^{39}$ Nowadays, rESWT devices are often used because they are less expensive than fESWT generators. Nonetheless, there is no conclusive evidence determining which type of ESWT, radial or focused, has more effective clinical utility in reducing muscle spasticity and improving motor function after stroke.

In the last five years, a few review studies have provided primary evidence to support the use of ESWT for the upper and lower limb spasticity: one meta-analysis of clinical trials on all types of spasticity in patients after brain injury, ${ }^{40}$ two meta-analyses of randomized controlled trials (RCTs) on spasticity in post-stroke patients, ${ }^{41,42}$ one narrative review on muscle hypertonia and dystonia, ${ }^{43}$ and one authorized narrative review on upper and lower limb spasticity in post-stroke patients. ${ }^{44}$ Additionally, several studies have confirmed utility of ESWT in reducing spasticity among patients with cerebral palsy (CP, 8 studies on the total sample of 124 patients, 4 used fESWT and 4 rESWT) ${ }^{45-52}$ and multiple sclerosis (MS, 1 study involved a group of 34 patients treated with rESWT). ${ }^{53}$

\section{Limited Data on Shock Waves}

The most recent systematic review and meta-analysis by Xiang et $\mathrm{al}^{42}$ included 8 RCTs that compared active ESWT with placebo ESWT on the sample of 385 patients with muscle spasticity after stroke. In this study, the ESWT was used as a monotherapy or as additional modality used in conjunction with pharmacotherapy and/or physical therapy as adjunctive management for post-stroke spasticity in adult patients. The results indicate that active ESWT is significantly superior to placebo ESWT, and this conclusion seems to be quite obvious. This study has several limitations such as significant heterogeneity in the metaanalysis, relatively small number of RCT subjects treated with ESWT, limited data to assess follow-up results of ESWT, and evaluation of spasticity based on limited clinical measurement methods like MAS and Modified Tardieu Scale (MTS). The meta-analysis mentioned earlier did not compare the rESWT and fESWT treatments directly.

An earlier meta-analysis by Guo et $\mathrm{al}^{41}$ has also shown that ESWT is useful for the treatment of spasticity in poststroke patients. However, the authors used the MAS as only one method for spasticity assessment and did not consider important factors that may potentially affect the results, such as ESWT frequency, therapy site, total ESWT sessions, and muscles subjected to ESWT sessions. 
Moreover, there was a lack of RCTs among the included articles, which may negatively affect the quality of this publication. This study also has some limitations, such as qualifying for analysis, a limited total number of six relevant articles with only the RCT protocol, the insufficient number of analyzed studies and patients in each of the study, and the presence of potential publication bias. Also, there were no comparisons done between both types of ESWT.

For these purposes, the authors recognize the importance of carrying out an up-to-date systematic review and qualitative synthesis to better assess the effectiveness of the ESWT and its effects on reducing muscle spasticity in post-stroke survivors. It is worth noting that thus far only one RCT study has directly compared the effects of fESWT versus rESWT applied with identical EFD for spasticity reduction. ${ }^{54}$

Therefore, the primary aim was to compare different types of ESWT (fESWT and rESWT) and their clinical efficiency for reduction of muscle spasticity in adult patients after stroke. The secondary aim was to conduct the evaluation of scientific reliability and methodological quality of recent clinical trials according to their level of evidence. And the tertiary aim was to highlight the importance of ESWT in current neurorehabilitation and to discuss further clinical and research implications in this area.

\section{Methods}

\section{Protocol and Registration}

The literature search was performed in May 2019. This paper was designed in accordance with the current guidelines of the Preferred Reporting Items for Systematic Reviews and Meta-Analyses - PRISMA $2009^{55}$ and PRISMA-P 2015. ${ }^{56}$ Moreover, the systematic review was prospectively registered in the PROSPERO database (International Prospective Register of Ongoing Systematic Reviews) with registration no. CRD42018105138. ${ }^{57,58}$

\section{Eligibility Criteria}

Only studies that met inclusion criteria were selected and used for further analysis. Title, abstract, and keywords were analyzed first, and then the full text was reviewed. To qualify, articles had to have appeared in peer-reviewed impact factor journals, have been published after the year 2000, and be available in the full-text, English-language version. No limitation was placed on ESWT type, treatment period, or measurement tools. Only studies including post-stroke etiology of spastic paresis were considered.

\section{Search Strategy}

A systematic search was performed using the PubMed, PEDro, Scopus, and EBSCOhost databases for studies on ESWT and post-stroke spasticity. The following research terms were used: "Muscle AND (Spastic OR Hypertonia) OR (Spasticity) AND Shock Wave AND Stroke" for PubMed database; "Stroke AND Spasticity AND Muscle AND Shock Wave" for PEDro database; "Stroke AND (Muscle OR Limb) AND (Spastic OR Hypertonia) OR (Spasticity) AND (Shock Wave OR ESW)" for Scopus database; and "Stroke AND Spastic OR Hypertonia OR Spasticity AND Shock Wave OR ESW" for EBSCOhost database. The current studies examining ESWT for patients with spasticity, the therapeutic efficacy of ESWT for patients with spastic paresis after stroke, and the relationship between obtained clinical effect and different setup parameters and protocols were investigated.

\section{Study Selection}

This review is based on studies that were published from the time each database was created through May 2019 and were selected after a computerized search strategy. All clinical trials that investigated the efficiency and safety of ESWT for muscle spasticity were initially included regardless of study design. All qualified studies were selected according to their methodological protocol as randomized-controlled trials (RCTs), clinical-controlled trials (CCTs), prospective-clinical trials (PCTs), and clinical case reports (CCRs). No limitations were placed on ESWT type, treatment period, measurement tools, or spasticity severity. The follow-up data were also considered from these studies. All literature reviews, editorial comments, conference reports, and letters to the editors were excluded.

\section{Data Extraction}

Assessment of papers' eligibility was performed by two independent reviewers (RD and JT) and, in the case of disagreement, the judgment of a third reviewer (MS) was sought. The most significant details of the study, including its protocol, patients' characteristics, clinical outcomes, assessment tools, follow-up period, ESWT parameters, ESWT-related adverse events, local anesthesia, and p-values, were abstracted manually (RD and JT) and the data were verified (MS). Additional significant data were 
analyzed as well, including PEDro quality score, level of evidence according to Sackett gradation, the journals' current impact factor, where the paper has been published, as well as a total sum of citations and average citations per year for each of the studies (RD and JT). Finally, the data were verified (MS).

\section{Synthesis of Results}

In total, in the first stage of this review, 118 records associated with the subject terms were identified from PubMed $(n=30)$, PEDro $(n=6)$, Scopus $(n=35)$, and EBSCOhost $(\mathrm{n}=47)$. In the screening process, duplicated studies were removed $(n=32)$ and papers judged improper on the basis of titles and abstracts $(n=49)$ were excluded.
Among 37 other records, a detailed review of full-text was conducted, and 20 more studies were rejected for not meeting the inclusion criteria.

Finally, 17 studies were included in a qualitative synthesis for a systematic review. The amplified version of the PRISMA flow chart of screening and selection results are presented in Figure 1. The papers were then divided by ESWT type - fESWT $(\mathrm{n}=10)$ and rESWT $(n=7)$ - used for the antispastic procedure to compare each technology in terms of selected clinical, technical and methodological parameters. A study by $\mathrm{Wu}$ et $\mathrm{al}^{54}$ was conducted using both fESWT and rESWT groups due to the fact that they compared these applications within the same RCT study.

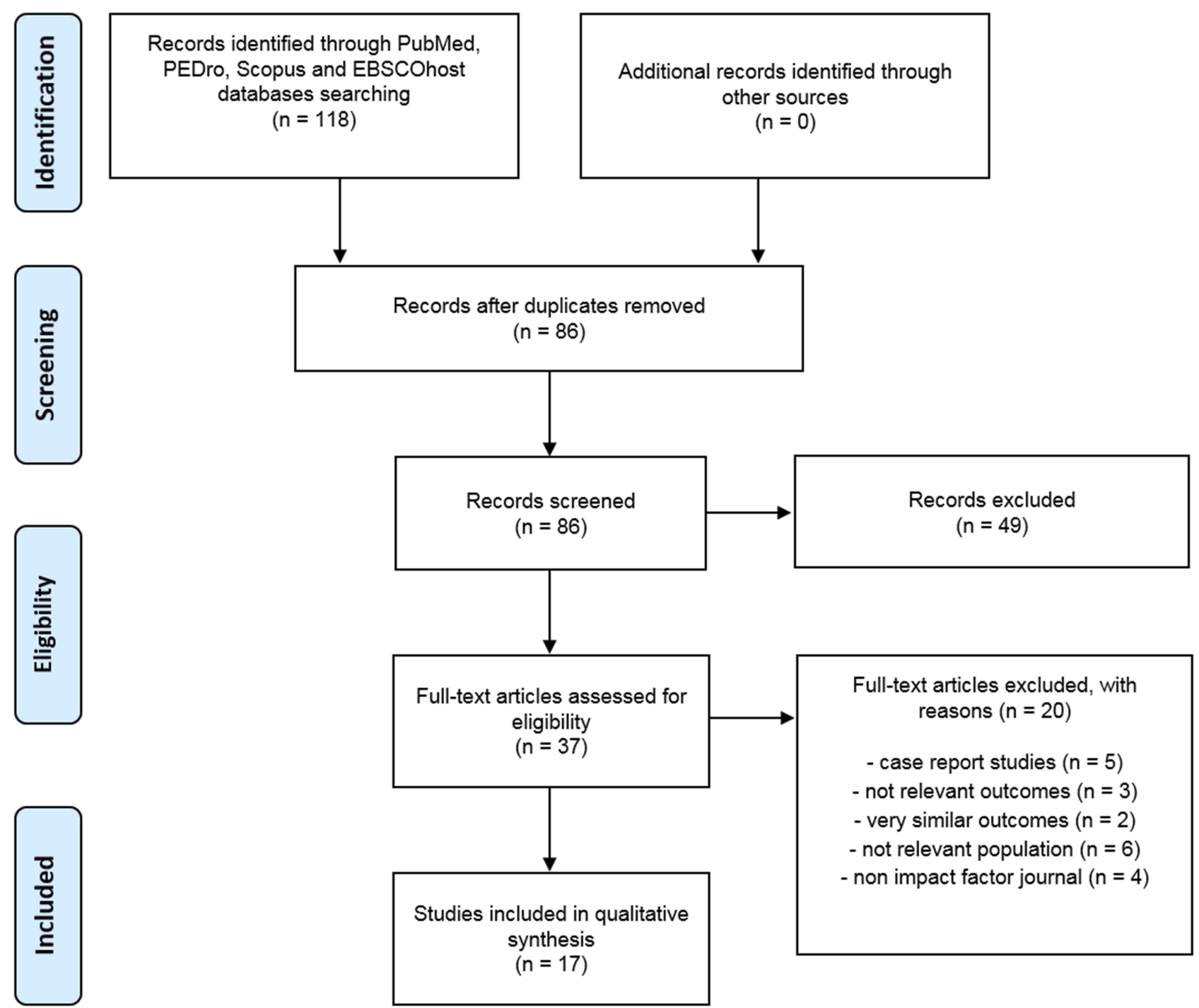

Figure I Flow chart of the studies selection process for the systematic review according to the PRISMA guidelines.

Abbreviations: PRISMA, Preferred Reporting Items for Systematic Reviews and Meta-Analyses; PubMed, National Library of Medicine; PEDro, Physiotherapy Evidence Database; EBSCO, Elton B. Stephens Company database; n, number. 


\section{Data Analysis}

For the purposes of the systematic review, spasticityrelated clinical outcomes were carefully analyzed: (1) the spasticity level was assessed with common tools such as Modified Ashworth Scale (MAS) or Modified Modified Ashworth Scale (MMAS); (2) passive range of motion (ROM) of the joints in goniometric measurement (GOM); and (3) the most common neurophysiological parameter $-\mathrm{H} / \mathrm{M}$ ratio $(\mathrm{H} / \mathrm{M}-\mathrm{R})$ to measure alpha-motor neuron excitability, which could be assessed by stretch reflex (particularly regarding spasticity) using surface electromyography (sEMG). Additionally, such parameters as motor nerve conduction velocity (MNC-V), compound motor action potential latency (CMAP-L), compound motor action potential amplitude (CMAPA), F wave latency (FW-L), and F wave amplitude (FW-A) were analyzed to assess motor neuron function due to the presence of any adverse signs caused by nerve lesioning.

Changes between each measurement were specified using percent as the value of delta percent $[\Delta \%]$ as a referral and comparable value for all assessed clinical outcomes. Important clinical information regarding the administration of the local anesthesia (LA) prior to the ESWT application, as well as any documentation of adverse events (AE) following ESWT, were analyzed.

The technical parameters of ESWT used during an intervention, as well as the most significant details of the treatment session, were also considered. All qualified papers were additionally classified by scientific reliability using the Physiotherapy Evidence Database (PEDro) quality assessment tool, which is a valid measure of the methodological quality of clinical trials, ${ }^{59}$ and the Sackett grade system for evaluation of the level of evidence according to with Evidence-Based Medicine (EBM) ${ }^{60}$

Data analysis was performed using Excel software (Microsoft, US) and Statistica (StatSoft, Dell Inc., US). Descriptive statistics for quantitative variables such as mean (M), standard deviation (SD), median (Me), and lower (Q1) and upper (Q3) quartiles were calculated. Comparison of results between fESWT group and rESWT group including primary clinical outcomes as AS/MAS, ROM, and H/R-M was carried out using the U-MannWhitney test (with continuity correction). Alpha level was set at 0.05 for all comparisons.

\section{Results}

\section{Outcome Measurements for Shock Waves}

For purposes of the systematic review, the selected clinical outcomes were analyzed and compared between the baseline (M0) and the first measurement time point after ESWT (M1). The following outcomes were analyzed: (1) spasticity level as $\Delta \%$ of grade in MAS or MMAS tools in 16 studies (nine studies in fESWT vs 7 studies in rESWT), ${ }^{54,61-74}$ (2) range of motion as $\Delta \%$ of degree in GOM assessment of nine studies (six studies in fESWT vs three studies in rESWT) ${ }^{54,63,64,67,68,70,72,75}$ and (3) selected electrophysiological parameters in such as MNC-V as $\Delta \%$ of ms, CMAP-L as $\Delta \%$ of ms, CMAP-A as $\Delta \%$ of $\mathrm{mV}$, FW-L as $\Delta \%$ of ms, FW-A as $\Delta \%$ of $\mu \mathrm{V}$, HR-L as $\Delta \%$ of ms and H/M-R as $\Delta \%$ in six studies (four studies in fESWT vs two studies in rESWT), ${ }^{65,67,70,73,75}$ It should be also noted that follow-up observations were undertaken in 12 studies within a mean period of 5.5 weeks ( 6.2 weeks in fESWT vs 4.7 weeks in rESWT) ${ }^{54,62-66,68,70,73}$ (Table 1).

\section{Summary of MAS Results}

The results for spasticity levels with MAS and MMAS in fESWT groups at baseline measurements (M0) were ranged between 2.22 and 4.10 grade ( $2.97 \pm 0.70$ grade) in comparison to results presented in the first measurement after fESWT application (M1) which varied from 1.22 to 2.70 grade ( $1.91 \pm 0.75$ grade). This resulted in a mean difference showing clinical improvement in spasticity between pre- and post-intervention at $\Delta=34.45 \% .{ }^{54,61-64,70-73}$ In turn, the spasticity level in rESWT groups at M0 was ranged between 1.53 and 3.30 grade $(2.29 \pm 0.59$ grade) in comparison to results presented after rESWT application varied from 0.73 to 2.30 (1.42 \pm 0.52 grade). This resulted in mean differences showing clinical improvement in spasticity between pre- and postinterventions at $\Delta=34.97 \%$. This comparison indicates the similar clinical effect in spasticity reduction for both ESWT modalities $(\Delta=0.52 \%)$, though slightly better for rESWT, however statistically non-significant for M0 $(\mathrm{p}=0.153)$, M1 $(\mathrm{p}=0.223)$, and M1-M0 $(\mathrm{p}=0.525)^{54,65-69,74}$ (Figure 2 and Table 1).

\section{Summary of ROM Results}

The results for ROM with GOM in fESWT groups at M0 were ranged between 9.90 and 56.67 of angular degrees (29.53 \pm 8.21 degrees) in comparison to results presented at M1 varied from 16.40 to 59.16 of angular degrees (37.37 
Table I Clinical Trials on fESWT and rESWT in Post-Stroke Muscles' Spasticity Including Clinical Outcomes and Additional Information

\begin{tabular}{|c|c|c|c|c|c|c|c|c|}
\hline No. & Type & Study & Muscles & Outcomes [Results*] & $\begin{array}{l}\text { FU** } \\
\text { [Weeks] }\end{array}$ & LA & $\mathbf{A E}$ & AT \\
\hline I. & fESWT & Manganotti \& Amelio $2005^{70}$ & $\mathrm{CF}$ and $\mathrm{IO}$ & MAS (+); ROM (+); EMG (-) & 12 & No & NS & NS \\
\hline 2. & & Santamato et al $2013^{61}$ & FDS & MAS (+); SFS (+); VAS $(+)$ & 12 & No & No & NS \\
\hline 3. & & Sohn et al $201 I^{71}$ & APF & MAS (+); EMG (-) & 0 & NS & NS & Yes \\
\hline 4. & & Moon et al $2013^{72}$ & APF & MAS (+); ROM (-); FMA $(-)$; IDT (+) & 4 & No & No & Yes \\
\hline 5. & & Santamato et al $2014^{73}$ & APF & $\operatorname{MAS}(+) ; \operatorname{ROM}(+) ;$ EMG $(-)$ & 4 & No & Yes & No \\
\hline 6. & & Sawan et al $2017^{75}$ & APF & EMG (+); ROM (+); I0-MWT (+) & 0 & NS & NS & Yes \\
\hline 7. & & Yoon et al $2017^{62}$ & $\mathrm{EF}$ or $\mathrm{KF}$ & MAS (+); MTS (+) & 4 & NS & No & Yes \\
\hline 8. & & Taheri et al $2017^{63}$ & APF & MAS $(+)$; VAS $(+)$; ROM $(+)$; 3-MWD $(+)$; LEFS $(+)$ & 12 & NS & NS & Yes \\
\hline 9. & & Wu et al $2017^{54}$ & APF & MAS $(+)$; MTS $(+)$; ROM $(+)$; I0-MWT (+); FPMP $(+)$ & 8 & NS & No & NS \\
\hline 10 & & Lee et al $2018^{64}$ & APF & MAS (+); ROM (+); FMA (+); USG (+) & 4 & NS & NS & Yes \\
\hline I. & rESWT & Daliri et al $2015^{65}$ & $\mathrm{CF}$ & MMAS $(+)$; BRS $(-)$; EMG $(+)$ & 5 & NS & NS & NS \\
\hline 2. & & Li et al $2016^{66}$ & $\mathrm{CF}$ & MAS (+); FMA (+) & 16 & No & No & Yes \\
\hline 3. & & Radinmehr et al $2016^{67}$ & APF & MMAS (+); ROM (+); PPFT (+); TUG (+); EMG (-) & 0 & NS & No & No \\
\hline 4. & & Kim et al $2016^{68}$ & APF & VAS (+); CMS (+); ROM (-); FMA (-); MAS (-) & 4 & No & Yes & NS \\
\hline 5. & & Dymarek et al $2016^{74}$ & $\mathrm{CF}$ & MAS (+); sEMG (+); IRT (+) & 0 & No & No & No \\
\hline 6. & & Dymarek et al $2016^{69}$ & $\mathrm{CF}$ & MAS (+); sEMG (+); IRT (+) & 0 & No & No & No \\
\hline 7. & & Wu et al $2017^{54}$ & APF & MAS (+); MTS (+); ROM (+); I0-MWT (+); FPMP (+) & 8 & NS & No & NS \\
\hline
\end{tabular}

Abbreviations: fESWT, focused shock wave therapy; rESWT, radial shock wave therapy; FU**, follow-up analysis at the last measurement time-point; LA, local anesthetics; AE, adverse events; AT, additional treatment during ESWT session; NS, not specified; CF, carpal flexors muscles; IO, interosseous muscles; FDS, flexor digitorum superficialis muscle; APF, ankle plantar flexors muscles; EF, elbow flexors muscles; KF, knee flexors muscles; MAS, Modified Ashworth Scale; MMAS, Modified Modified Ashworth Scale; VAS, Visual-Analogue Scale; ROM, range of motion; I0-MWT, I0-meter walk test; 3-MWD, 3-meter Walk durationd TUG, Timed UP and Go test; LEFS, Lower Extremity Functional Score; MTS, Modified Tardieu Scale; FMA, Fugl-Meyer Assessment; SFS, Spasm Frequency Scale; BRS, Brunnstrom Recovery Stage; CMS, Constant-Murley Score; IDT, Isokinetic Dynamometer Testing; EMG, electromyography; sEMG, surface electromyography; IRT, infrared thermal imaging; FPMP, foot pressure measurement platform; USG, ultrasonography; PPFT, passive plantarflexor torque; $(+)$, ESWT significantly better statistically than either placebo or alternative treatment modalities or pretreatment; (-), ESWT not significantly better statistically than either placebo or alternative treatment modalities or pre-treatment; results*significantly better statistically improvement between pre- and Ist post-treatment measurement time-point.

\pm 7.77 degrees), giving a mean difference of clinical improvement in ROM between pre- and post-intervention at $\Delta=38.83 \%$ of angular degrees. ${ }^{54,63,64,70,72,75}$ ROM values in rESWT groups at M0 were ranged between 20.30 and 82.85 of angular degrees $(42.78 \pm 11.81)$; results presented at M1 varied from 28.00 to 87.23 of angular degrees (51.31 \pm 11.88 degrees), giving a mean difference of clinical improvement in ROM between pre- and postintervention at $\Delta=32.26 \%$. This comparison determines the more beneficial effect of fESWT in the enhancement of ROM compared to rESWT $(\Delta=6.57 \%)$, nevertheless statistically non-significant for $\mathrm{M} 0(\mathrm{p}=0.897), \mathrm{M} 1(\mathrm{p}=0.897)$, and M1-M0 ( $\mathrm{p}=0.897)^{54,67,68}$ (Figure 3 and Table 1).

\section{Summary of Neurophysiological Results}

The results for H/M-R in EMG examination in fESWT groups at M0 were ranged between $2.93 \%$ and $5.63 \%$ $(4.28 \pm 1.77 \%)$ in comparison to results presented at M1 varied from $1.79 \%$ to $5.76 \%(3.78 \pm 1.26 \%)$, giving a mean difference of clinical improvement in H/M-R between preand post-intervention at $\Delta=18.32 \%$. H/M-R values in
rESWT groups at M0 were ranged between $0.45 \%$ and $2.40 \%$ (1.38 $1.09 \%)$; results presented at M1 varied from $0.41 \%$ to $1.48 \%(0.95 \pm 0.50 \%)$, giving a mean difference of clinical improvement in H/M-R between pre- and postintervention at $\Delta=22.27 \%$. This comparison determines the more beneficial effect of rESWT in the enhancement of $\mathrm{H} /$ M-R compared to fESWT $(\Delta=3.95 \%)$, which suggests a limited trend in improving alpha motor neuron excitability, at a statistically insignificant level for M0 $(\mathrm{p}=0.245)$, M1 ( $\mathrm{p}=0.245)$, and M1-M0 ( $\mathrm{p}=0.699) .{ }^{65,67,71,75}$

The consolidated mean results for both fESWT and rESWT groups in electrophysiological evaluation at M0 vs M1 were $51.85 \pm 4.52 \mathrm{~ms}$ vs $52.61 \pm 4.28 \mathrm{~ms}$ for MNC-V (difference at $\Delta=1.40 \%$ ); $3.36 \pm 0.86 \mathrm{~ms}$ vs $3.83 \pm 0.97 \mathrm{~ms}$ for CMAP-L (difference at $\Delta=5.56 \%$ ); $11.28 \pm 2.83 \mathrm{mV}$ vs $11.30 \pm 2.80 \mathrm{mV}$ for CMAP-A (difference at $\Delta=0.15 \%$ ); $36.17 \pm 3.76 \mathrm{~ms}$ vs $35.49 \pm 3.09 \mathrm{~ms}$ for FW-L (difference at $\Delta=2.71 \%$ ); $700 \pm 100 \mu \mathrm{V}$ vs $650 \pm 200 \mu \mathrm{V}$ for FW-A (difference at $\Delta=7.14 \%$ ) - all the differences listed above were non-significant which indicates noninvasive character of ESWT (Figure 4 and Table 1). 


\section{MAS/MMAS MO \\ $\mathbf{p}=\mathbf{0 . 1 5 3}$}

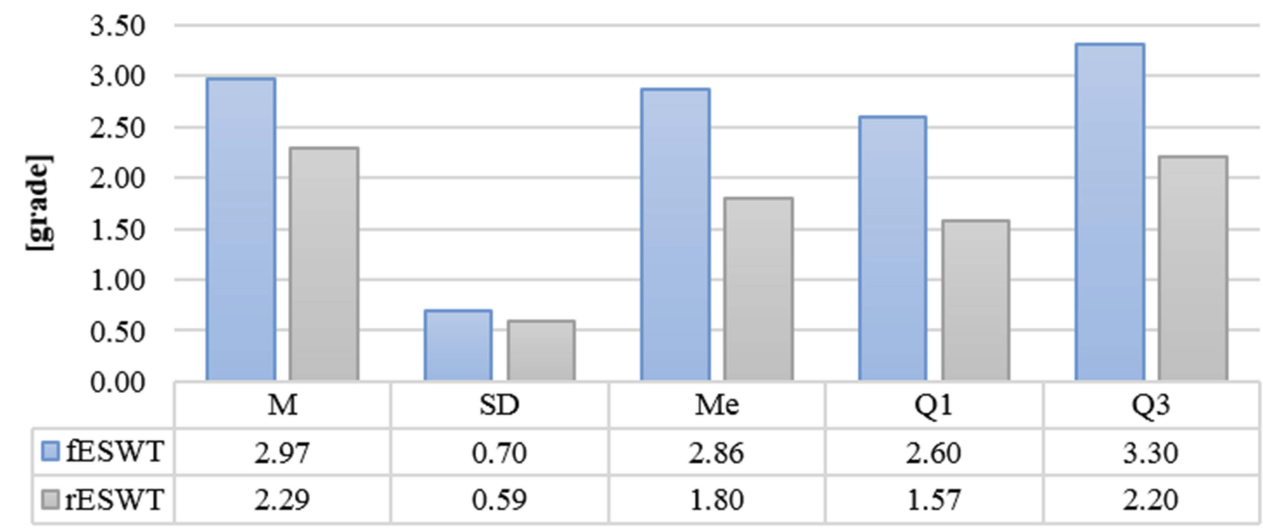

\section{MAS/MMAS M1}

$$
\mathbf{p}=\mathbf{0 . 2 2 3}
$$

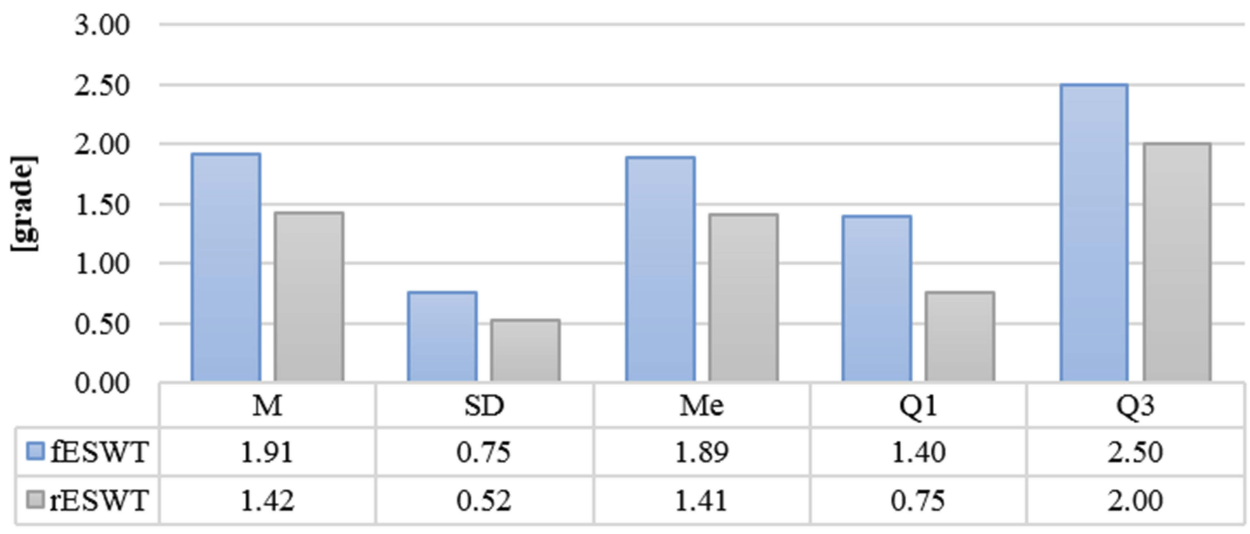

\section{MAS/MMAS M1-M0}
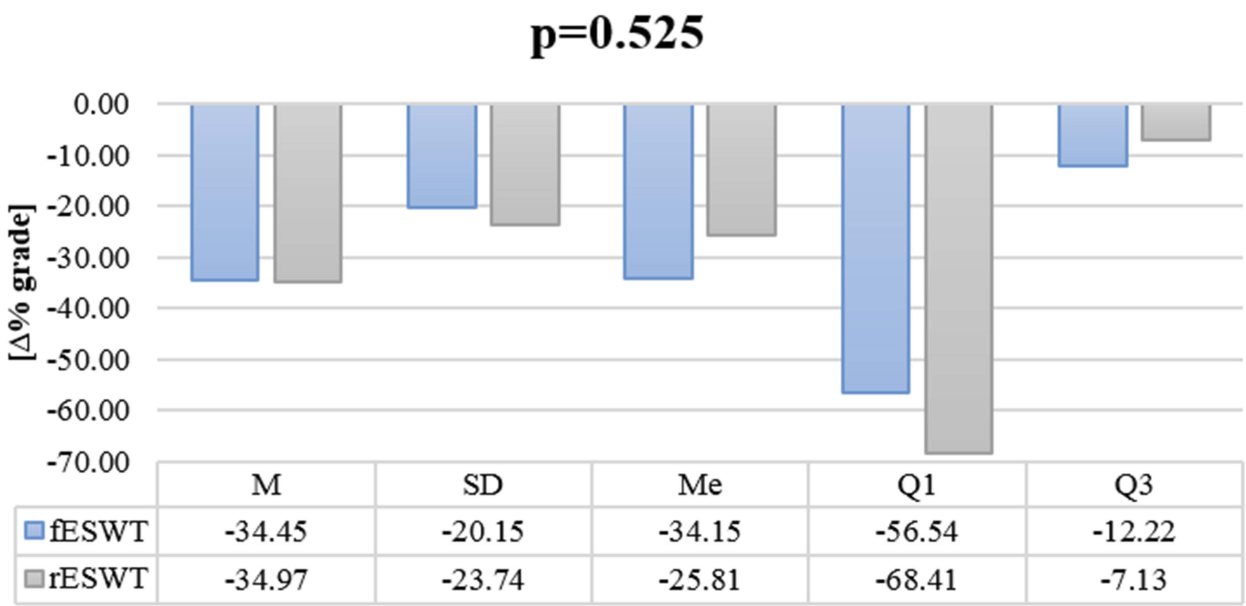

Figure 2 Summary of results for spasticity levels with MAS or MMAS.

Abbreviations: fESWT, focused shock wave therapy; rESWT, radial shock wave therapy; MAS, Modified Ashworth Scale; MMAS, Modified Modified Ashworth Scale; M0, pre-ESWT measurement; MI, post-ESWT measurement; M, mean; SD, standard deviation; Me, median; QI, Ist quartile; Q3, 3rd quartile. 


\section{ROM M0}

$\mathrm{p}=\mathbf{0 . 8 9 7}$

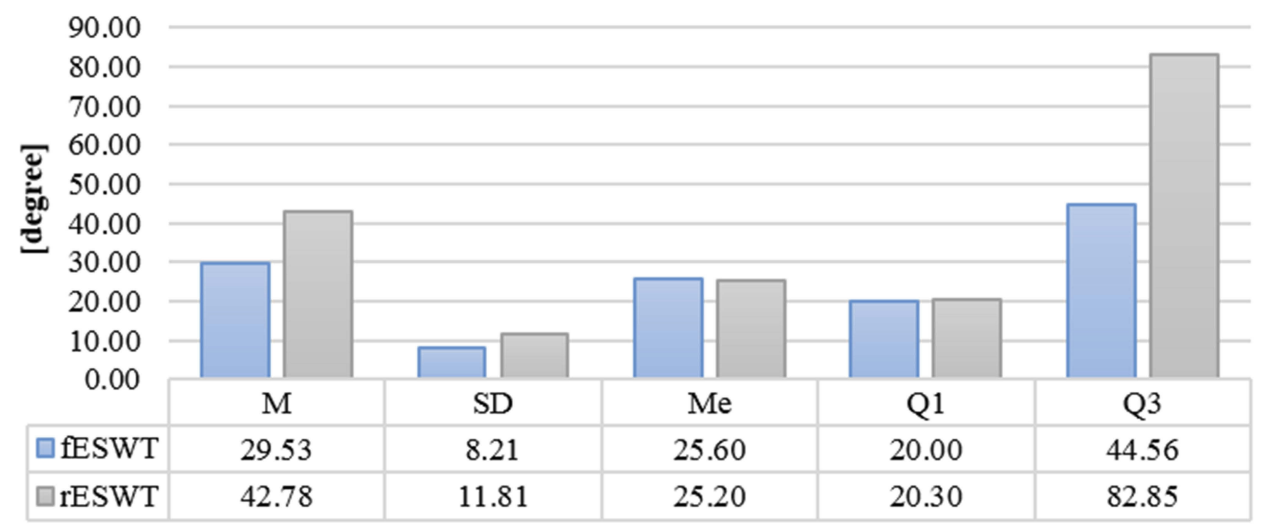

ROM M1

$\mathbf{p}=\mathbf{0 . 8 9 7}$

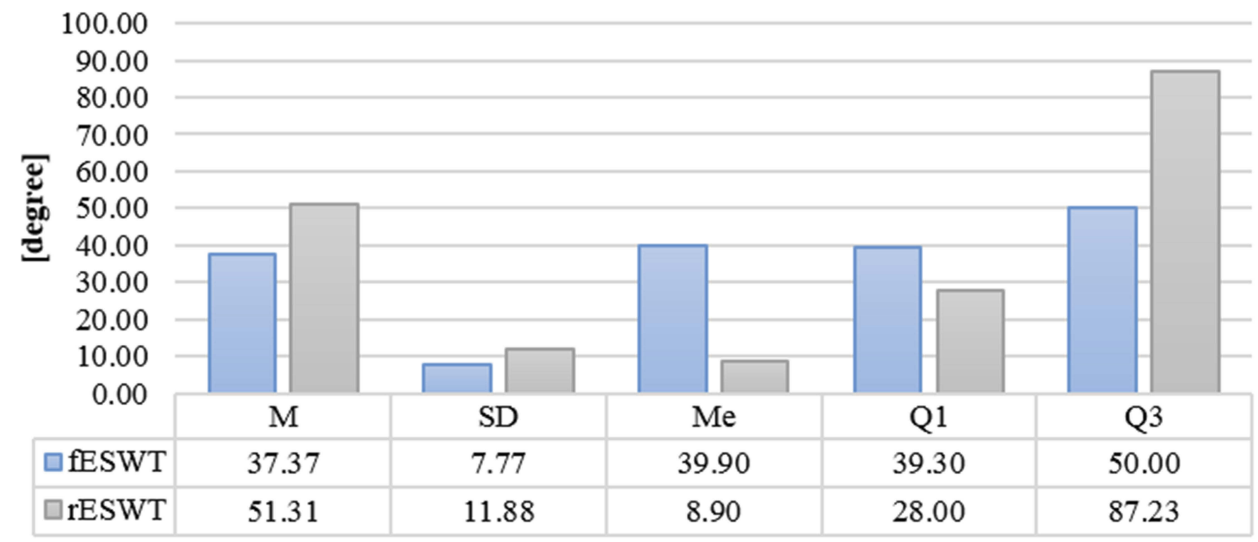

ROM M1-M0

$\mathbf{p}=\mathbf{0 . 8 9 7}$

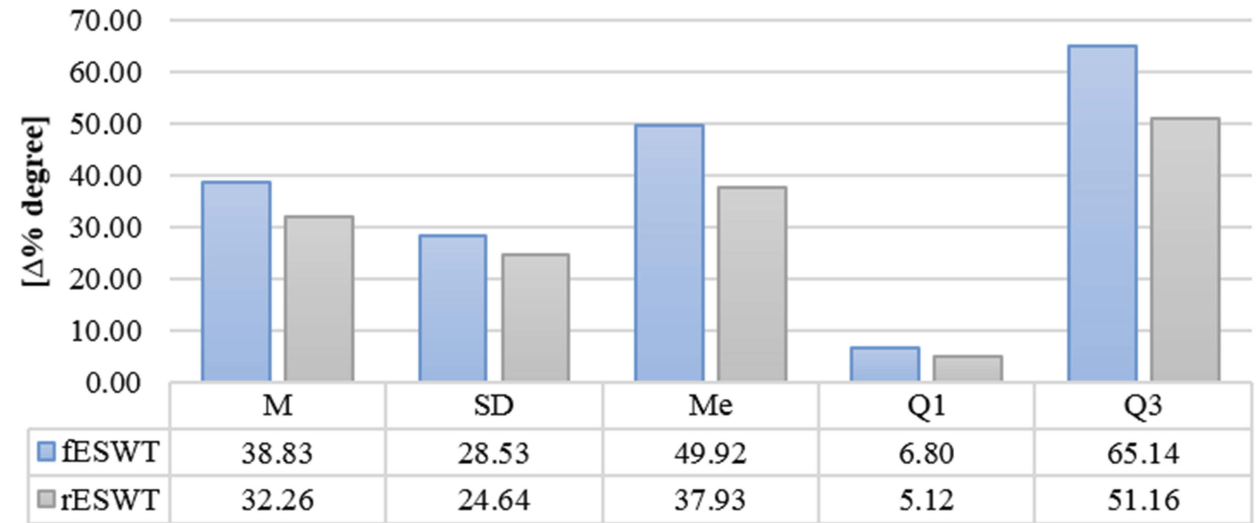

Figure 3 Summary of results for ROM with GOM.

Abbreviations: fESWT, focused shock wave therapy; rESWT, radial shock wave therapy; ROM, range of motion; GOM, goniometric measurement; MO, pre-ESWT measurement; MI, post-ESWT measurement; M, mean; SD, standard deviation; Me, median; QI, Ist quartile; Q3, 3rd quartile. 


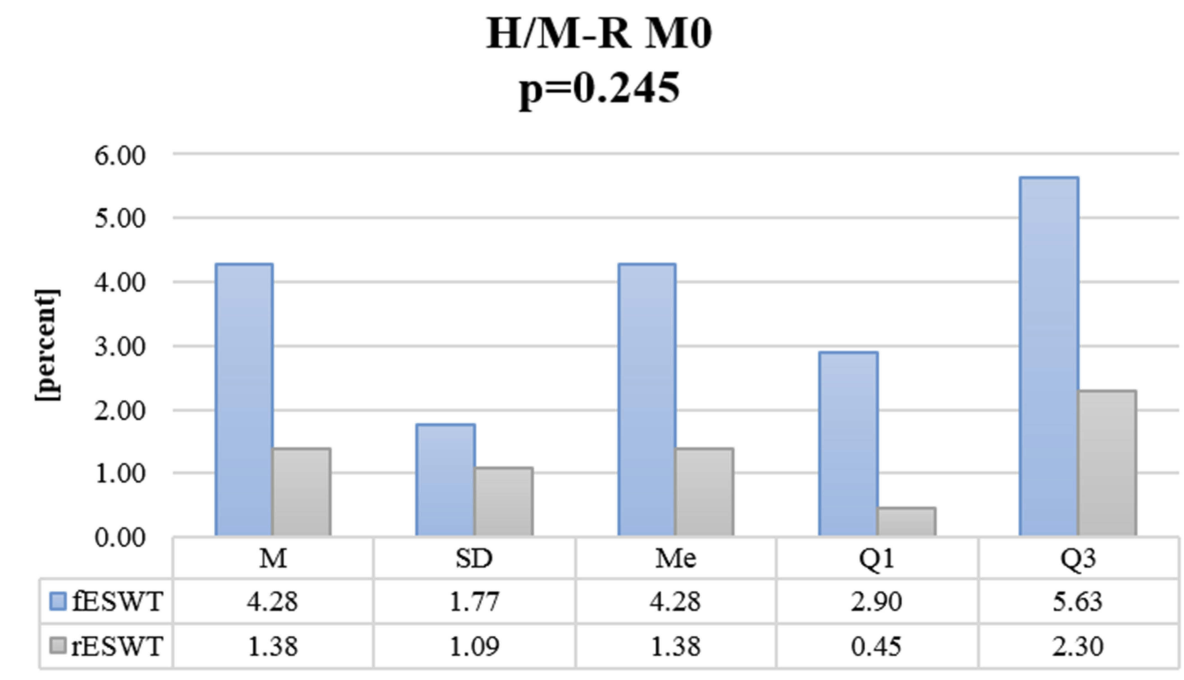

\section{H/M-R M1}

$\mathrm{p}=\mathbf{0 . 2 4 5}$

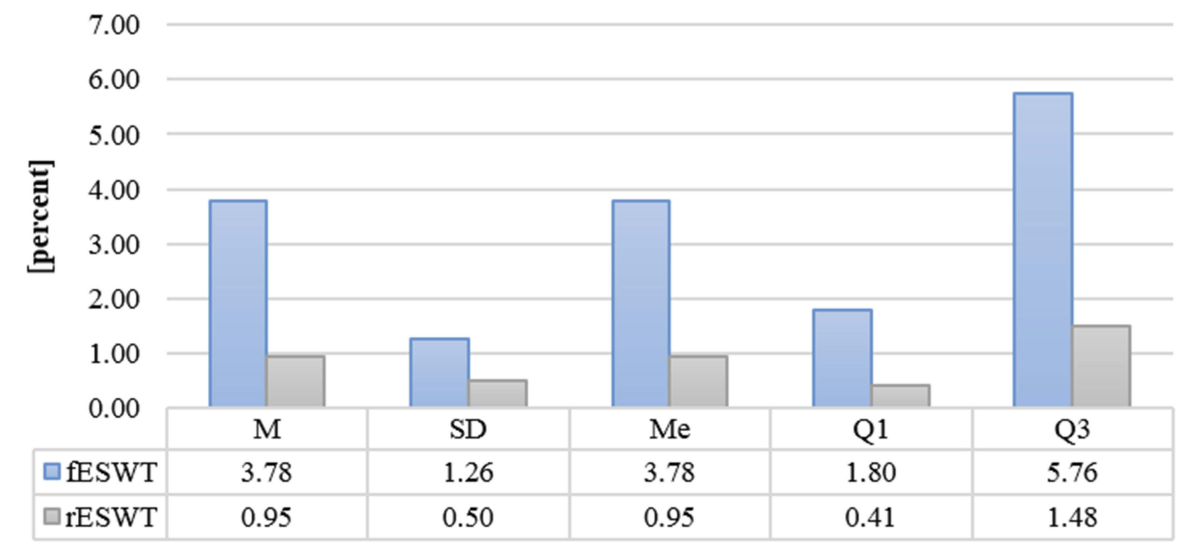

H/M-R M1-M0

$\mathrm{p}=\mathbf{0 . 6 9 9}$

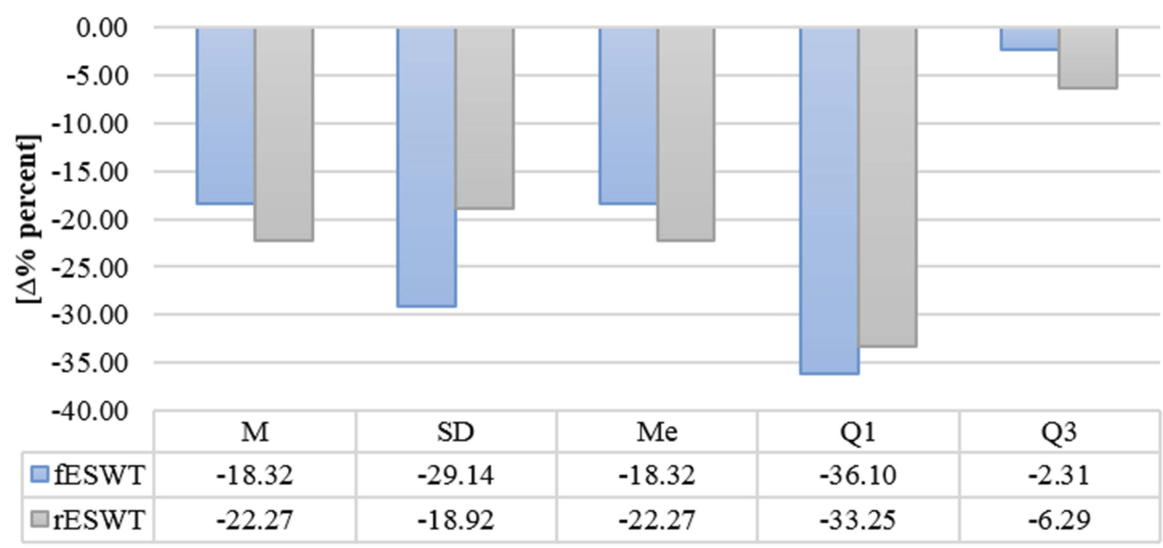

Figure 4 Summary of results for H-M/R with EMG.

Abbreviations: fESWT, focused shock wave therapy; rESWT, radial shock wave therapy; H/M-R, H/M ratio; EMG, Electromyography; M0, pre-ESWT measurement; MI, post-ESWT measurement; M, mean; SD, standard deviation; Me, median; QI, Ist quartile; Q3, 3rd quartile. 


\section{Local Anesthetics and Side Effects}

It was found that in the ESWT groups the local anesthetic transcutaneous injections were not performed in eight studies. ${ }^{61,66,68-70,72-74}$ This clinically important issue was not specified in the case of nine articles $54,62-65,67,71,75$ (Table 1).

Only 11 cases of unexpected ESWT-related side effects were identified (pain $n=5$, lower limb muscular weakness $n=2$, petechiae at the treatment site $n=3$, and small bulla $\mathrm{n}=1) .{ }^{67,73}$ It should be noted that all side effects were tolerated quite well by all patients and were resolved in a few days. Unfortunately, there were studies with lacking information about the presence or absence of any adverse events $^{62-64,69,70,72}$ (Table 1).

\section{Studies' Characteristics and Additional Therapies}

According to the design of the study protocols, 11 of the 17 qualified studies were represented by the RCTs with the highest reliability of research data (five in fESWT vs six in rESWT), ${ }^{54,61-69}$ four were CCTs without randomized allocation (four in fESWT vs zero in rESWT), ${ }^{70-72,75}$ two were PCTs without controls (one in fESWT vs one in rESWT), ${ }^{73,74}$ and there were no CCRs (Table 2).

Additional treatment was continued in seven of the studies. ${ }^{62-64,66,71,72,75}$ During the studies, patients continued receiving antispastic pharmacological therapy and did not change the dose of antispastic medication, ${ }^{62,64,66,71,72}$ ROM exercises, stretching, and physical therapy. ${ }^{85,93}$ The physiotherapy program consisted of resistance and balance exercises, gait and functional training, and supported by the use of ankle-foot orthosis, ${ }^{75}$ as well as oral antispastic medications and stretching exercises, which were similar for both groups. ${ }^{63}$ Antispastic medication and rehabilitation programs remained unchanged from two months before the study to the end of the follow-up period ${ }^{66}$ (Table 1).

\section{Characteristics of Patients Treated with Shock Waves}

A total of 17 studies $^{54,61-75}$ involving a sample of 303 patients (182 patients in fESWT vs 130 patients in rESWT) were included in this systematic review. The mean number of $18.35 \pm 6.24$ patients was presented per study (18.20 \pm 6.86 patients in fESWT vs $18.57 \pm 5.77$ patients in rESWT). The gender distribution was 116 female patients (65 in fESWT vs 51 in rESWT) and 196 male patients (117 in fESWT vs 79 in rESWT). The mean age was $57.87 \pm 10.45$ years $(55.89 \pm 10.84$ years in fESWT vs $59.84 \pm 10.05$ years in rESWT) and the mean duration from the onset of stroke was $40.49 \pm 25.63$ months $(36.34$ \pm 25.61 months in fESWT vs $44.64 \pm 25.65$ months in rESWT). The etiology of spasticity in all 17 studies was a chronic stroke. Among all patients, 198 experienced

Table 2 Clinical Trials on fESWT and rESWT in Post-Stroke Muscles' Spasticity Including Patients' Characteristics

\begin{tabular}{|c|c|c|c|c|c|c|c|}
\hline No. & Type & Study & Country & Design & Patients [n] & Age [years] & Duration [months] \\
\hline $\mathrm{I}$. & fESWT & Manganotti \& Amelio $2005^{70}$ & Italy & CCT & 20 & $63.0(38 \div 76)$ & NS \\
\hline 2. & & Santamato et al $2013^{61}$ & Italy & RCT & 16 & $64.4 \pm 6.09$ & $10.5 \pm 12.2$ \\
\hline 3. & & Sohn et al $201 I^{71}$ & Korea & ССТ & 10 & $44.9 \pm 11.3$ & $53.4 \pm 23.9$ \\
\hline 4. & & Moon et al $2013^{72}$ & Korea & СCT & 30 & $52.6 \pm 14.9$ & $2.5 \pm 1.5$ \\
\hline 5. & & Santamato et al $2014^{73}$ & Italy & PCT & 23 & $57.6 \pm 10.8$ & $24.9 \pm 11.9$ \\
\hline 6. & & Sawan et al $2017^{75}$ & Egypt & СCT & 40 & $50.6 \pm 6.7$ & NS \\
\hline 7. & & Yoon et al $2017^{62}$ & Korea & RCT & 80 & $58.70 \pm 17.50$ & $100.30 \pm 98.30$ \\
\hline 8. & & Taheri et al $2017^{63}$ & Iran & RCT & 13 & $56.50 \pm 11.60$ & $33 \pm 21.4$ \\
\hline 9. & & Wu et al $2017^{54}$ & Taiwan & RCT & 15 & $60.30 \pm 9.90$ & $53.2 \pm 26.7$ \\
\hline 10 & & Lee et al $2018^{64}$ & Korea & RCT & 9 & $50.89 \pm 8.81$ & $11.89 \pm 8.99$ \\
\hline I. & rESWT & Daliri et al $2015^{65}$ & Iran & RCT & 15 & $54.4 \pm 9.4$ & $30.0 \pm 22.5$ \\
\hline 2. & & $\mathrm{Li}$ et al $2016^{66}$ & China & RCT & 20 & $55.35 \pm 3.05$ & $61.70 \pm 9.73$ \\
\hline 3. & & Radinmehr et al $2016^{67}$ & Iran & RCT & 12 & $59.0 \pm 13.0$ & $34.3 \pm 20.6$ \\
\hline 4. & & Kim et al $2016^{68}$ & Korea & RCT & 17 & $65.88 \pm 8.27$ & $26.11 \pm 33.08$ \\
\hline 5. & & Dymarek et al $2016^{74}$ & Poland & РCT & 20 & $63.15 \pm 12.60$ & $43.35 \pm 42.11$ \\
\hline 6. & & Dymarek et al $2016^{69}$ & Poland & RCT & 30 & $61.43 \pm 12.74$ & $51.30 \pm 25.46$ \\
\hline 7. & & Wu et al $2017^{54}$ & Taiwan & RCT & 16 & $59.60 \pm 11.30$ & $55.70 \pm 26.10$ \\
\hline
\end{tabular}

Abbreviations: $\mathrm{fESWT}$, focused shock wave therapy; rESWT, radial shock wave therapy; $\mathrm{n}$, number; RCT, randomized controlled trial; CCT, clinical controlled trial; PCT, prospective clinical trial; NS, not specified. 
ischemic (101 patients in fESWT vs 97 patients in rESWT) and 114 had a hemorrhagic stroke (81 patients in fESWT vs 33 patients in rESWT) ${ }^{54,61-75}$ (Table 2).

Among the ten papers in which the fESWT was used for spasticity treatment, the application involved muscles of the upper extremity in two studies ${ }^{61,70}$ and the lower extremity in eight. ${ }^{54,62,64,71-73,75}$ Among seven papers with rESWT, the application involved muscles of the upper extremity in the case of four studies ${ }^{65,66,69,74}$ and the lower extremity in three. ${ }^{54,67,68}$ The most frequently treated muscles with fESWT were the ankle plantar flexor muscles $^{54,63,64,71-73,75}$ and with rESWT were the carpal flexors ${ }^{65,66,69,74}$ (Table 1).

\section{Antispastic Sessions and Parameters of Shock Waves}

In the ESWT groups, the mean number of treatment sessions was three (range of 1-6 sessions in fESWT vs $1-8$ sessions in rESWT). The mean interval between ESWT sessions was six days (range of 3-7 days in fESWT vs 1-7 days in rESWT). The mean ESWT treatment period was characterized by a large impurity, and it was 12 days (range of 1-42 days in fESWT vs 1-21 days in rESWT). ${ }^{54,61-75}$ The most common type of fESWT generators was electromagnetic, ${ }^{54,61-64,70,73}$ and the most useful manufacturer of fESWT devices was STORZ Medical (Switzerland). ${ }^{54,61,68,70}$ All seven studies used pneumatic generators for rESWT sessions ${ }^{54,65-69,74}$ and the most useful manufacturer of rESWT devices was BTL Ind. (United Kingdom) (Table 3).

In the fESWT groups, the mean number of shockwave pulses of each treatment was $1882 \pm 553$ (same range of 1500-3000). The mean energy flux density (EFD) was 0.1 $\mathrm{mJ} / \mathrm{mm}^{2}\left(0.08 \pm 0.03 \mathrm{~mJ} / \mathrm{mm}^{2}\right.$ in fESWT vs $0.11 \pm 0.1 \mathrm{~mJ} / \mathrm{mm}^{2}$ in rESWT). The mean total energy density (TED, calculated as the product of the number of sessions, the number of pulses, and the EFD) was $0.48 \pm 0.48 \mathrm{~J} / \mathrm{mm}^{2}(0.36 \pm 0.28 \mathrm{~J} /$ $\mathrm{mm}^{2}$ in fESWT vs $\left.0.64 \pm 0.64 \mathrm{~J} / \mathrm{mm}^{2}\right)$. The mean pressure was $2 \pm 0.52$ bars $(1.8 \pm 0.25$ bars in fESWT vs $2.11 \pm 0.73$ bars in rESWT). The mean frequency was $5 \pm 2.16 \mathrm{~Hz}(4.3 \pm 0.52$ $\mathrm{Hz}$ in fESWT vs $5.6 \pm 2.88 \mathrm{~Hz}$ in rESWT) ${ }^{54,61-75}$ (Table 4).

In the RCT studies that contain a placebo group, the following methods for blinding the ESWT interventions were used: sham treatments were provided with the same equipment by only receiving a sound without any transducer contact with the skin; ${ }^{62,64}$ placebo interventions were carried out with no energy applied; ${ }^{65,66}$ with removed transducer; ${ }^{68}$ or using a special polyethylene cup filled as a mechanical barrier $^{69}$ (Table 4).

\section{PEDro Assessment and Studies' Quality}

Assessment of methodological quality was performed using the PEDro scale with a proper Sackett gradation. The PEDro

Table 3 Clinical Trials on fESWT and rESWT in Post-Stroke Muscles' Spasticity Including the Type of Device and Therapy Period

\begin{tabular}{|c|c|c|c|c|c|c|c|}
\hline No. & Type & Study & Type & Device [Company, Country] & $\mathbf{S}[\mathrm{n}]$ & I [n] & $\mathbf{P}[\mathbf{d}]$ \\
\hline I. & fESWT & Manganotti \& Amelio $2005^{70}$ & EM & Modulith SLK (Storz Medical, SUI) & 1 & 0 & I \\
\hline 2. & & Santamato et al $2013^{61}$ & EM & Minilith SLI (Storz Medical, SUI) & 3 & 3 & 9 \\
\hline 3. & & Sohn et al $2011^{71}$ & $\mathrm{EH}$ & Evotronn (SwiTech, SUI) & 1 & 0 & I \\
\hline 4. & & Moon et al $2013^{72}$ & $\mathrm{PE}$ & PiezoWave (Richard Wolff, GER) & 3 & 7 & 21 \\
\hline 5. & & Santamato et al $2014^{73}$ & EM & EvoTron RFL0300 (Sanuwave AG, SUI) & 1 & 0 & 1 \\
\hline 6. & & Sawan et al $2017^{75}$ & NS & NS & 6 & 7 & 42 \\
\hline 7. & & Yoon et al $2017^{62}$ & EM & Dornier Aries (Dornier MedTech, GER) & 3 & 7 & 21 \\
\hline 8. & & Taheri et al $2017^{63}$ & EM & Dornier AR2 (Dornier MedTech, GER) & 3 & 7 & 21 \\
\hline 9. & & Wu et al $2017^{54}$ & EM & Duolith SDI (Storz Medical, SUI) & 3 & 7 & 21 \\
\hline 10. & & Lee et al $2018^{64}$ & EM & Dornier Aries (Dornier MedTech, GER) & 1 & 0 & 1 \\
\hline I. & rESWT & Daliri et al $2015^{65}$ & $\mathrm{PN}$ & NS model (BTL Ind., UK) & 1 & $\mathrm{n}$ & I \\
\hline 2. & & Li et al $2016^{66}$ & PN & Physio SWT (Pagani Elettronica, ITA) & 3 & 7 & 21 \\
\hline 3. & & Radinmehr et al $2016^{67}$ & PN & enPuls 2.0 (Zimmer Medizin Syst., GER) & I & $\mathrm{n}$ & 1 \\
\hline 4. & & Kim et al $2016^{68}$ & PN & Masterpuls MP200 (Storz Medical, SUI) & 8 & 1.5 & 14 \\
\hline 5. & & Dymarek et al $2016^{74}$ & PN & BTL-5000 SWT (BTL Ind., UK) & 1 & $\mathrm{n}$ & 1 \\
\hline 6. & & Dymarek et al $2016^{69}$ & PN & BTL-5000 SWT (BTL Ind., UK) & 1 & $\mathrm{n}$ & I \\
\hline 7. & & Wu et al $2017^{54}$ & $\mathrm{PN}$ & Duolith SDI (Storz Medical, SUI) & 3 & 7 & 21 \\
\hline
\end{tabular}

Abbreviations: fESWT, focused shock wave therapy; rESWT, radial shock wave therapy; S, ESWT sessions; I, interval between ESWT sessions; $n$, number; P, period of ESWT; d, days; EM, electromagnetic device; EH, electrohydraulic device; PE, piezoelectric device; PN, pneumatic device; NS, not specified; SUI, Switzerland; GER, Germany; UK, United Kingdom; ITA, Italy. 
Table 4 Clinical Trials on fESWT and rESWT in Post-Stroke Muscles' Spasticity Including Treatment Parameters and Sham Interventions

\begin{tabular}{|c|c|c|c|c|c|c|c|c|}
\hline No. & Type & Study & Pulses [n] & $\mathbf{P}[$ Bar $]$ & $\begin{array}{l}\text { EFD } \\
{\left[\mathrm{mJ} / \mathrm{mm}^{2}\right]}\end{array}$ & $\begin{array}{l}\text { TED } \\
{\left[\mathrm{J} / \mathrm{mm}^{2}\right]}\end{array}$ & $F[\mathrm{~Hz}]$ & Sham ESWT \\
\hline I. & fESWT & Manganotti \& Amelio $2005^{70}$ & 15003200 & 1.5 & 0.03 & 0.07 & NS & None \\
\hline 2. & & Santamato et al $2013^{61}$ & 2000 & 1.5 & 0.10 & 0.06 & 4 & None \\
\hline 3. & & Sohn et al $201 I^{71}$ & 1500 & 2.0 & 0.10 & 0.15 & NS & None \\
\hline 4. & & Moon et al $2013^{72}$ & 1500 & 2.0 & 0.09 & $0.4 I$ & 4 & None \\
\hline 5. & & Santamato et al $2014^{73}$ & 1500 & 1.5 & 0.03 & 0.05 & NS & None \\
\hline 6. & & Sawan et al $2017^{75}$ & 1500 & NS & NS & NS & NS & None \\
\hline 7. & & Yoon et al $2017^{62}$ & 1500 & 0.1 & 2.0 & 0.45 & 5 & Sound, non-contact \\
\hline 8. & & Taheri et al $2017^{63}$ & 1500 & 0.1 & 2.0 & 0.45 & 4 & Sound, no energy \\
\hline 9. & & Wu et al $2017^{54}$ & 3000 & 0.1 & 2.0 & 0.90 & 5 & None \\
\hline 10. & & Lee et al $2018^{64}$ & 2000 & 0.1 & 2.0 & 0.20 & 4 & Sound, non-contact \\
\hline I. & rESWT & Daliri et al $2015^{65}$ & 1500 & 1.5 & 0.03 & 0.05 & 4 & Sound, no energy \\
\hline 2. & & Li et al $2016^{66}$ & 2750 & 3.3 & 0.20 & 1.65 & 5 & None \\
\hline 3. & & Radinmehr et al $2016^{67}$ & 2000 & 3.0 & 0.30 & 0.60 & 5 & None \\
\hline 4. & & Kim et al $2016^{68}$ & 1500 & 2.0 & 0.10 & 1.20 & 12 & Removed transducer \\
\hline 5. & & Dymarek et al $2016^{74}$ & 1500 & 1.5 & 0.03 & 0.05 & 4 & Polyethylene barrier \\
\hline 6. & & Dymarek et al $2016^{69}$ & 1500 & 1.5 & 0.03 & 0.05 & 4 & None \\
\hline 7. & & Wu et al $2017^{54}$ & 3000 & 2.0 & 0.10 & 0.90 & 5 & None \\
\hline
\end{tabular}

Abbreviations: fESWT, focused shock wave therapy; rESWT, radial shock wave therapy; $n$, number; P, pressure; EFD, energy flux density; TED, total energy density; F, frequency; $\mathrm{Hz}$, Hertz; NS, not specified.

scale was developed by the Physiotherapy Evidence Database to determine the quality of clinical trials. These tools are reliable and valid for rating the quality of research studies. ${ }^{76}$ Studies scoring 9-10 on the PEDro scale were considered methodologically to be of "excellent" quality and studies scoring 6-8 were considered "good" quality. Studies in these two categories received Level 1 of evidence in the Sackett's grading system. Studies scoring from 4 to 5 were qualified as "fair" quality and studies scoring less than 4 qualified as "poor" quality; studies in these categories received Level 2 of evidence in Sackett's grading system ${ }^{77}$ (Table 5). The mean PEDro score among studies on ESWT was $5.12 \pm 2.27(4.7 \pm 2.5$ in fESWT vs $5.71 \pm 2.21$ in rESWT). The highest Level 1 of evidence was given to three of the ten studies in fESWT which give $33.3 \%{ }^{54,61,64}$ versus four of the seven studies in rESWT $-57 \%{ }^{54,66,68,69}$ (Table 5).

\section{Impact Factor and Bibliometric}

\section{Assessment}

Regarding the values of current journal IF (indicating to some extent the prestige of the publishing journal in the international arena and determining the value of the study), the mean score was $2.179 \pm 0.923(2.295 \pm 1.534$ in fESWT and $2.064 \pm 0.313$ in rESWT). It should be noted that IF value was comparable for both ESWTs, though slightly higher for
fESWT studies. In turn, the values of 5-year journal IF, the mean score was $2.534 \pm 1.057(2.784 \pm 1.919$ in fESWT and $2.284 \pm 0.230$ in rESWT). Of the fESWT papers, three studies have not been found in the Web of Science database because there is a lack of 5-year IF for the journal of Annals of Rehabilitation Medicine, and thus they have not been included for this analysis, ${ }^{62,71,72}$ and of the rESWT papers, there was only one paper also published in the Journal of Annals of Rehabilitation Medicine ${ }^{68}$ (Table 6).

A total sum of citations and average number of citations per year of the papers (showing the potential scientific value of each paper and presenting a real interest of the researchers onto obtained results) were also compared for fESWT and rESWT based on Web of Science Core Collection by Thomson Reuters. A mean value of total and average citations for fESWT was 19 and 2.05 \pm 2.02 , respectively. The same mean value for rESWT was 2 and $0.67 \pm 0.82$, respectively (Table 6 ).

\section{Discussion}

To the best of our knowledge, this study is the first worldwide systematic review with qualitative synthesis comparing both types of ESWT used for reduction of spastic paresis and for improvement of motor recovery in hemiplegic patients after stroke. The main findings of our 
Table 5 Clinical Trials on fESWT and rESWT in Post-Stroke Muscles' Spasticity Including Their Quality Evaluation Based on PEDro Assessment Criteria

\begin{tabular}{|c|c|c|c|c|c|c|c|c|c|c|c|c|c|c|c|}
\hline \multirow[t]{2}{*}{ No. } & \multirow[t]{2}{*}{ Type } & \multirow[t]{2}{*}{ Study } & \multicolumn{12}{|c|}{ PEDro Assessment Criteria } & \multirow[t]{2}{*}{ EL } \\
\hline & & & $I^{*}$ & 2 & 3 & 4 & 5 & 6 & 7 & 8 & 9 & 10 & II & TS & \\
\hline I. & fESWT & Manganotti \& Amelio $2005^{70}$ & + & - & - & + & - & - & - & + & - & + & + & $4 / 10$ & 2 \\
\hline 2. & & Santamato et al $2013^{61}$ & + & + & - & + & - & - & + & + & - & + & + & $6 / 10$ & I \\
\hline 3. & & Sohn et al $2011^{71}$ & + & - & - & - & - & - & - & + & - & - & + & $2 / 10$ & 2 \\
\hline 4. & & Moon et al $2013^{72}$ & + & - & - & + & - & - & - & + & - & + & + & $4 / 10$ & 2 \\
\hline 5. & & Santamato et al $2014^{73}$ & + & - & - & - & - & - & - & - & - & - & + & $1 / 10$ & 2 \\
\hline 6. & & Sawan et al $2017^{75}$ & + & - & - & - & - & - & - & + & - & + & + & $3 / 10$ & 2 \\
\hline 7. & & Yoon et al $2017^{62}$ & + & + & - & + & - & - & - & + & - & + & + & $5 / 10$ & 2 \\
\hline 8. & & Taheri et al $2017^{63}$ & + & + & - & + & - & - & - & + & - & + & + & $5 / 10$ & 2 \\
\hline 9. & & Wu et al $2017^{54}$ & + & + & + & + & + & - & + & + & - & + & + & $8 / 10$ & 1 \\
\hline 10. & & Lee et al $2018^{64}$ & + & + & + & + & + & + & + & + & - & + & + & $9 / 10$ & 1 \\
\hline I. & rESWT & Daliri et al $2015^{65}$ & + & - & - & + & + & - & - & - & - & + & + & $4 / 10$ & 2 \\
\hline 2. & & Li et al $2016^{66}$ & + & + & - & + & + & - & + & + & + & + & + & $8 / 10$ & I \\
\hline 3. & & Radinmehr et al $2016^{67}$ & + & + & - & - & + & + & - & - & - & + & + & $5 / 10$ & 2 \\
\hline 4. & & Kim et al $2016^{68}$ & + & + & - & + & + & - & + & + & - & + & + & $7 / 10$ & I \\
\hline 5. & & Dymarek et al $2016^{74}$ & + & - & - & - & - & - & - & + & - & - & + & $2 / 10$ & 2 \\
\hline 6. & & Dymarek et al $2016^{69}$ & + & + & - & + & + & - & - & + & - & + & + & $6 / 10$ & I \\
\hline 7. & & Wu et al $2017^{54}$ & + & + & + & + & + & - & + & + & - & + & + & $8 / 10$ & I \\
\hline
\end{tabular}

Notes: Legend for PEDro criteria: I - Eligibility criteria were specified; 2 - Subjects were randomly allocated to groups; 3 - Allocation was concealed; 4 - The groups were similar at baseline regarding the most important prognostic indicators; 5 - There was blinding of all subjects; 6 - There was blinding of all therapists who administered the therapy; 7 - There was blinding of all assessors who measured at least one key outcome; 8 - Measures of at least one key outcome were obtained from $>85 \%$ of the subjects initially allocated to groups; 9 - All subjects for whom outcome measures were available received the treatment or control condition as allocated or, where this was not the case, data for at least one key outcome were analyzed by "intention to treat"; 10 - The results of between-group statistical comparisons are reported for at least one key outcome; II - The study provides both point measures and measures of variability for at least one key outcome.

Abbreviations: fESWT, focused shock wave therapy; rESWT, radial shock wave therapy; TS, total PEDro score by Physiotherapy Evidence Database; EL, evidence level (Sackett's grading system); PEDro, Physiotherapy Evidence Database; I*, eligibility criteria item does not contribute to the total score.

systematic review present detailed information about the outcome measurements and selected results analyzed for pre- and post-ESWT time-points focusing on the most important clinical outcomes (endpoints) such as spasticity level, range of motion and neurophysiological parameters, which have been assessed in each of the studies using the same or similar and relevant research tools. The supplementary finding includes characteristics of patients treated with ESWT, parameters of ESWT for the antispastic procedure, the presence of side effects after ESWT, the methodological quality of ESWT studies with their level of evidence, and examples of bibliographic data indicating the utility of ESWT studies for the international research community.

\section{Main Findings}

According to the most frequently assessed and most typical clinical outcomes for post-stroke spasticity, a total of 15 studies with total of 283 patients evaluated the effects of ESWT on MAS or MMAS grades pre- and post-intervention, 8 studies with total 143 patients measured ROM with goniometry. The first and most important observation is that all studies analyzed in this review show an unambiguous and statistically significant improvement in terms of both the reduction of spasticity and relaxation of the muscles studied, as well as the enhancement in ROM and larger angular degrees in the joints studied. Our detailed comparison demonstrated a similar clinical effect in spasticity reduction for both ESWT types, with a slightly better effect for $\operatorname{rESWT}(\Delta=0.52 \%)$, statistically insignificant $(\mathrm{p}<0.525)$. The situation is different in the case of ROM, where a better improvement has been demonstrated for fESWT ( $\Delta=6.57 \%$ ), also without statistical significance $(\mathrm{p}=0.897)$. However, the limitations of AS and MAS tools in measuring spasticity should be remembered, especially according to their qualitative and subjective information, concerning validity and reliability, lack of standardization, not well-controlled stimulus, and also lack of their reliability and validity for all muscle groups. ${ }^{78,79}$ The validity and reliability of these scales are not without controversy and probably these impressiveness affected the obtained negative correlation between AS/MAS and ROM on the basis of analyzed data from 15 clinical studies. Moreover, both results are 
Table 6 Clinical Trials on fESWT and rESWT in Post-Stroke Muscles' Spasticity Including Their Quality Evaluation Based on The Web Of Science Core Collection Statistics

\begin{tabular}{|c|c|c|c|c|c|c|}
\hline No. & Type & Study & Journal & IF & TC & AC \\
\hline I. & fESWT & Manganotti \& Amelio $2005^{70}$ & Stroke & 6.239 & 65 & 4.64 \\
\hline 2. & & Santamato et al $2013^{61}$ & Ultrasound in Med. \& Biol. & 2.645 & 18 & 3.00 \\
\hline 3. & & Sohn et al $20 \mathrm{II}^{71}$ & Ann Rehabil Med. & 1.681 & NF & $\mathrm{NF}$ \\
\hline 4. & & Moon et al $2013^{72}$ & Ann Rehabil Med. & 1.681 & NF & NF \\
\hline 5. & & Santamato et al $2014^{73}$ & Top Stroke Rehabil. & I.77I & 13 & 2.60 \\
\hline 6. & & Sawan et al $2017^{75}$ & Neuro-Rehabilitation & 1.495 & NF & NF \\
\hline 7. & & Yoon et al $2017^{62}$ & Ann Rehabil Med. & 1.681 & NF & $\mathrm{NF}$ \\
\hline 8. & & Taheri et al $2017^{63}$ & Arch Iran Med. & 1.254 & 0 & 0.00 \\
\hline 9. & & Wu et al $2017^{54}$ & Eur J Phys Rehab Med. & 2.208 & 0 & 0.00 \\
\hline 10. & & Lee et al $2018^{64}$ & $P M \& R$ & 1.85 & NF & $\mathrm{NF}$ \\
\hline I. & rESWT & Daliri et al $2015^{65}$ & Neuro-Rehabilitation & 1.779 & 8 & 2.00 \\
\hline 2. & & $\mathrm{Li}$ et al $2016^{66}$ & Medicine & 2.028 & 3 & 1.00 \\
\hline 3. & & Radinmehr et al $2016^{67}$ & Disabil Rehabil. & 2.042 & 2 & 1.00 \\
\hline 4. & & Kim et al $2016^{68}$ & Ann Rehabil Med. & 1.681 & $\mathrm{NF}$ & $\mathrm{NF}$ \\
\hline 5. & & Dymarek et al $2016^{74}$ & Evid Based Complement Alternat Med. & 2.064 & 0 & 0.00 \\
\hline 6. & & Dymarek et al $2016^{69}$ & Ultrasound in Med. \& Biol. & 2.645 & 0 & 0.00 \\
\hline 7. & & Wu et al $2017^{54}$ & Eur J Phys Rehab Med. & 2.208 & 0 & 0.00 \\
\hline
\end{tabular}

Abbreviations: fESWT, focused shock wave therapy; rESWT, radial shock wave therapy; IF, impact factor (current); TC, total citations by Web of Science Core Collection; AC, average citations per year by Web of Science Core Collection; NF, not found.

without statistical significance for fESWT and rESWT groups and could be within the bounds of the measurement error. On the other hand, the most recent meta-analysis revealed satisfactory inter- and intra-rater agreement for MAS and better reliability for measurements of upper limbs than lower. ${ }^{80}$

According to the most important neurophysiological data, only 5 studies with a total of 100 patients tested neurophysiological parameters with EMG assessment, of which 4 have assessed H/M-R parameter. It should be emphasized that recent studies have not confirmed another potential antispastic mechanism resulting from ESWT treatments: the modification and reduction of muscle tone and tendons jerks by a decrease of spinal excitability in neurophysiological examinations of the spastic muscles. It is reported that the effect of ESWT on spinal excitability can be excluded as the main mechanism. However, our analyses demonstrated beneficial effect of rESWT $(\Delta=22.27 \%)$ in the enhancement of H/M-R compared to fESWT $(\Delta=18.32 \%$ with a difference of $\Delta=3.95 \%$ ), which suggests a limited trend in improving alpha motor neuron excitability, statistically non-significant $(p=699)$. Moreover, all the differences in motor nerve conduction were non-significant showing lack of changes in the amplitude or latency of distal motor action potential and late responses, which indicates noninvasive character of ESWT without any episodes of neuromuscular denervation as a potential ESWT-related adverse effect.
Previous studies using electrophysiological data did not confirm the effect of ESWT on the spinal excitability underlying the stretch reflex and at the same time confirmed previous results obtained in patients with $\mathrm{CP}$ and MS treated with ESWT. However, it is well known that most of the clinical assessment tools, such as MAS, are not able to discriminate components between reflex and non-reflex hypertonia. These findings confirm the theory that ESWT influences the rheological properties of spastic muscles. Future research should focus specifically on more relevant assessments such as (1) ultrasonography for muscle fascicle length (MFL) and muscle thickness (MT); (2) real-time sonoelastography for red pixel intensity (RPI) to measure muscle stiffness by indirectly assessing the increased collagen content in spastic muscle; and (3) the novel technique of myotonometry that provides objective assessment by quantifying tissue displacement and examining the viscoelastic properties of spastic muscles.

\section{Supplementary Findings}

So far, more studies have been conducted with fESWT $(n=10)$ than with rESWT $(n=7)$. This is due to the fact that the use of fESWT has begun two decades earlier in general clinical practice, also in the case of muscle spasticity. Nevertheless, despite the substantial difference in the period, a slightly higher number of RCT protocol tests were 
conducted for rESWT $(n=6)$ as compared to fESWT $(n=5)$, which may indicate a better methodological quality of these assessments and more reliable results obtained.

The fact is that in previous studies a higher total number of patients was recruited for fESWT $(n=182)$, compared to rESWT $(n=130)$. However, the average number of patients per study is similar for both types of ESWTs and is slightly above 18 patients per each study, which argues in favor of almost the same target group of study participants. Also, the essential characteristics of patients were similar for fESWT and rESWT, such as mean age (55.89 vs 59.84 years), ischemic etiology of stroke (101 vs 97 patients), which indicates the homogeneity of the compared groups according to the ESWT type. Only mean duration from the onset of stroke was different between fESWT and rESWT (36.34 vs 44.64 months); however, patients in both groups were in the chronic period of stroke, where the muscle spasticity becomes fully developed, even established.

With regard to the extremities which have been treated with ESWT, the ankle plantar flexor muscles were the most frequently treated with fESWT, in turn, the carpal flexors were treated most frequently muscles with rESWT. This comparison indicates differences in the choice of patients' limbs subjected to ESWT sessions, but at the same time, it supports the clinical utility for both the upper and lower limb muscles. Regarding the presence of any ESWT-related side effects, only seven were observed after fESWT and four in case of rESWT. Fortunately, they were well tolerated by patients and disappeared spontaneously after a few days (pain, weakness, petechiae, bulla's).

It should be noted that the range of ESWT sessions was similar for fESWT (1-6 sessions) and rESWT (1-8 sessions). Even though treatments with fESWT are generally recommended once a week, and rESWT at intervals of 2-3 days, a similar interval between fESWT and rESWT sessions (3-7 vs 1-7 days) was observed. Also, all ESWT parameters for antispastic procedure were almost analogic for fESWT and rESWT: number of pulses per each treatment (1825 vs 1964 pulses) with range of pulses per each treatment (1500-3000 for both types of ESWT), energy flux density (0.08 vs $0.11 \mathrm{~mJ} / \mathrm{mm} 2)$; pressure (1.8 vs 2.1 bars). Only the frequency used was slightly lower for fESWT than for rESWT (4.3 vs $5.6 \mathrm{~Hz})$. This overview indicates the use of similar treatment parameters for spastic muscles, regardless of the type of ESWT used.

The highest Level 1 of evidence was given to three of the ten studies in fESWT and four of the seven studies in
rESWT. It should be noted that the best scores were obtained for criterion no. 1 (eligibility criteria were specified) which was presented in all 17 studies; however, this item did not contribute to the total PEDro score accordingly with instructions; and criterion no. 11 (both point measures and measures of variability for at least one key outcome were provided) presented in all studies, as well. A few criteria earned good scores and were presented on more than half of the studies: no. 2 (subjects were randomly allocated to groups), no. 4 (the groups were similar at baseline regarding the most important prognostic indicators), no. 8 (measures of at least one key outcome were obtained from $>85 \%$ of the subjects initially allocated to groups), and no. 10 (the results of between-group statistical comparisons are reported for at least one key outcome). In turn, the worst scores were observed for criterion no. 9 (data for at least one key outcome were analyzed by "intention to treat"), and no. 6 (there was blinding of all therapists who administered the therapy).

Articles for both fESWT and rESWT were published in journals of similar current (2.295 vs 2.064) and 5-year IF (2.784 vs 2.284). The analysis of bibliometric data shows that several interesting studies have been published over the last five years about the application of ESWT in spasticity. The average and total numbers of citations were drastically better for fESWT $(2.05 \pm 2.02$ and 19) than for $\operatorname{rESWT}(0.67 \pm 0.82$ and 2$)$. This difference is probably a consequence of the fact that the fESWT papers were published much earlier than those of the rESWT and two of them were somewhat pioneering articles in this field, which is they are cited higher in later publications of other researchers. We found that eight of ten papers with fESWT and all seven papers with rESWT have been published after the year 2013. That is why, in our opinion, a little longer time is needed to obtain better statistics on the quotations of these articles.

\section{Study Limitations}

There are a few limitations of this study that should be addressed. First and foremost, this study is a systematic review based on a comprehensive literature search; thus, more extensive analysis should be performed, such as meta-analysis of RCTs to determine ESWT utility and to demonstrate which type of ESWT is more effective in reduction of muscle spasticity. Second, non-English publications (Korean, Chinese, and Turkish), publications without quantitative data specified $(\mathrm{M} \pm \mathrm{SD})$, and publications listed outside the top database engines (KoreaMed, 
Index Copernicus, and Clarivate Analytics) were not included. Therefore, the language of the studies was limited to English, journals were limited to those with current IF, which may have resulted in bias. In addition, future review studies should also consider using the minimally clinically important difference (MCID) and/or minimal detectable change (MDC) which are critical for judging whether ESWT interventions have resulted in real change and the magnitude of the benefit perceived by the patient.

Subsequently, clinical conditions where spasticity coexisted with comorbid pathologies after stroke (knee osteoarthritis, heterotopic ossification, and plantar fasciitis) were not considered. Finally, qualitative synthesis of methodological issues was made using the PEDro scale; however, the risk of bias of RCTs studies could be extended by conducting an independent assessment in accordance to the guidelines for systematic reviews presented by Cochrane Collaboration Group. It should also be mentioned that the review consisted of studies performed in multiple countries from different regions of the world with different health-care systems and neurorehabilitation standards. Nevertheless, it seems that in the case of ESWT studies, the outcomes are generalizable and globally applicable.

\section{Conclusion}

Based on this systematic review, while the majority of current international studies are preliminary in nature with limited methodological design and moderate levels of evidence, it can be concluded that both fESWT and rESWT treatments are promising. Both are non-invasive and safe modalities for reducing muscle spasticity and improving motor recovery after stroke. Comparatively, rESWT may have a slightly better effect on the spasticity, and studies with rESWT are of higher methodological quality and level of evidence. Further well-planned clinical trials based on multicenter, prospective, placebo-controlled and RCTs are warranted, considering improvements of methodological imperfections. In the authors' opinion, it is time to utilize the success of ESWT to shed light on the practical guidelines that should be included in standard neurorehabilitation programs.

In the light of existing knowledge, an effort should be made to develop preliminary protocols or guidelines by the competent bodies, such as the World Federation for NeuroRehabilitation (WFNR), which associates specialists in neurological rehabilitation. The Cochrane Rehabilitation section from the Cochrane Collaboration, which first disseminates the available Cochrane evidence within the rehabilitation community, might also contribute to the development of such significant guidelines.

\section{Abbreviations}

$\mathrm{AE}$, adverse events; CCRs, clinical case reports; CCTs, clinical-controlled trials; CMAP-A, compound motor action potential amplitude; CMAP-L, compound motor action potential latency; CNS, central nervous system; $\mathrm{CP}$, cerebral palsy; EBM, evidence-based medicine; EFD, energy flux density; EMG-NMS, electromyography-triggered neuromuscular stimulation; ESWT, extracorporeal shock wave therapy; FES, functional electrical stimulation; fESWT, focused extracorporeal shock wave therapy; FW-A, F wave amplitude; FW-L, F wave latency; GOM, goniometric measurement; H/M-R, $\mathrm{H} / \mathrm{M}$ ratio; ISMST, International Society for Medical Shockwave Treatment; LA, local anesthesia; M, mean; MAS, Modified Ashworth Scale; Me, median; MFL, muscle fascicle length; MMAS, Modified Modified Ashworth Scale; MNC-V, motor nerve conduction velocity; MS, multiple sclerosis; MT, muscle thickness; MTS, Modified Tardieu Scale; NCS, neural stem cells; NMS, neuromuscular stimulation; NO, nitric oxide; NSC, neural stem cells; NTH-3, neurotrophin-3; PCTs, prospective-clinical trials; PEDro, Physiotherapy Evidence Database; Q1, 1st quartile; Q3, 3rd quartile; RCTs, randomized controlled trials; rESWT, radial extracorporeal shock wave therapy; ROM, range of motion; RPI, red pixel intensity; sEMG, surface electromyography; SD, standard deviation; tDCS, transcranial direct current stimulation; TED, total energy density; TENS, transcutaneous electrical nerve stimulation; TMS, transcranial magnetic stimulation; UMN, upper motoneuron; VEGF, vascular endothelial growth factor; WFNR, World Federation for NeuroRehabilitation.

\section{Funding}

This review study is theoretical part of the project conducted under a Research Grant for Young Scientists founded by the Ministry of Science and Higher Education of Poland from statutory sources of the Wroclaw Medical University in Poland (no. STM.E025.17.018) and supported by statutory grant of the Wroclaw Medical University in Poland for maintaining research potential (no. SUB. E020.19.003).

\section{Disclosure}

The authors report no conflicts of interest in this work. 


\section{References}

1. Li S. Spasticity, motor recovery, and neural plasticity after stroke. Front Neurol. 2017;8:120. doi:10.3389/fneur.2017.00120

2. Weerd LD, Rutgers WA, Groenier KH, Meer KVD. Perceived wellbeing of patients one year post stroke in general practice - recommendations for quality aftercare. BMC Neurol. 2011;11(1):42. doi:10.1186/1471-2377-11-42

3. Yelnik AP, Simon O, Parratte B, Gracies JM. How to clinically assess and treat muscle overactivity in spastic paresis. J Rehabil Med. 2010;42(9):801-807. doi:10.2340/16501977-0613

4. Lance J. Symposium Synopsis. In: Feldmann RG, Young RR, Koella WP, editors. Spasticity: Disordered Motor Control. Chicago: Year Book Medical Publishers; 1980:485-495.

5. Thibaut A, Chatelle C, Ziegler E, Bruno M-A, Laureys S, Gosseries O. Spasticity after stroke: physiology, assessment and treatment. Brain Inj. 2013;27(10):1093-1105. doi:10.3109/02699052.2013.804202

6. Trompetto C, Marinelli L, Mori L, et al. Pathophysiology of spasticity: implications for neurorehabilitation. Biomed Res Int. 2014;2014:354906. doi:10.1155/2014/354906

7. Li S, Shin H, Zhou P, Li X. Different effects of cold stimulation on reflex and non-reflex components of poststroke spastic hypertonia. Front Neurol. 2017;8:169. doi:10.3389/fneur.2017.00169

8. Foran JRH, Steinman S, Barash I, Chambers HG, Lieber RL. Structural and mechanical alterations in spastic skeletal muscle. Dev Med Child Neurol. 2005;47(10):713-717. doi:10.1017/S0012162205001465

9. Lieber RL, Steinman S, Barash IA, Chambers H. Structural and functional changes in spastic skeletal muscle. Muscle Nerve. 2004;29(5):615-627. doi:10.1002/mus.20059

10. Wu C-H, Ho Y-C, Hsiao M-Y, Chen W-S, Wang T-G. Evaluation of post-stroke spastic muscle stiffness using shear wave ultrasound elastography. Ultrasound Med Biol. 2017;43(6):1105-1111. doi:10.1016/j.ultrasmedbio.2016.12.008

11. Ward AB. A literature review of the pathophysiology and onset of post-stroke spasticity. Eur J Neurol. 2012;19(1):21-27. doi:10.1111/ j.1468-1331.2011.03448.x

12. Lundström E, Smits A, Borg J, Terént A. Four-fold increase in direct costs of stroke survivors with spasticity compared with stroke survivors without spasticity: the first year after the event. Stroke J Cereb Circ. 2010;41(2):319-324. doi:10.1161/STROKEAHA.109.558619

13. Marciniak C. Poststroke hypertonicity: upper limb assessment and treatment. Top Stroke Rehabil. 2011;18(3):179-194. doi:10.1310/ tsr1803-179

14. Bhimani RH, Anderson LC, Henly SJ, Stoddard SA. Clinical measurement of limb spasticity in adults: state of the science. $J$ Neurosci Nurs J Am Assoc Neurosci Nurses. 2011;43(2):104-115. doi:10.1097/ JNN.0b013e31820b5f9f

15. Smania N, Gandolfi M, Paolucci S, et al. Reduced-intensity modified constraint-induced movement therapy versus conventional therapy for upper extremity rehabilitation after stroke: a multicenter trial. Neurorehabil Neural Repair. 2012;26(9):1035-1045. doi:10.1177/ 1545968312446003

16. Good DC, Bettermann K, Reichwein RK. Stroke rehabilitation. Contin Minneap Minn. 2011;17(3Neurorehabilitation):545-567. doi:10.1212/ 01.CON.0000399072.61943.38

17. Veerbeek JM, van Wegen E, van Peppen R, et al. What is the evidence for physical therapy poststroke? A systematic review and meta-analysis. PLoS One. 2014;9:2. doi:10.1371/journal.pone.0087987

18. Notarnicola A, Moretti B. The biological effects of extracorporeal shock wave therapy (ESWT) on tendon tissue. Muscles Ligaments Tendons J. 2012;2(1):33-37.

19. Dymarek R, Halski T, Ptaszkowski K, Slupska L, Rosinczuk J, Taradaj J. Extracorporeal shock wave therapy as an adjunct wound treatment: a systematic review of the literature. Ostomy Wound Manage. 2014;60(7):26-39.
20. Wang C-J. Extracorporeal shockwave therapy in musculoskeletal disorders. J Orthop Surg. 2012;7:11. doi:10.1186/1749-799X-7-11

21. Speed C. A systematic review of shockwave therapies in soft tissue conditions: focusing on the evidence. Br J Sports Med. 2014;48 (21):1538-1542. doi:10.1136/bjsports-2012-091961

22. Foldager CB, Kearney C, Spector M. Clinical application of extracorporeal shock wave therapy in orthopedics: focused versus unfocused shock waves. Ultrasound Med Biol. 2012;38(10):1673-1680. doi:10.1016/j.ultrasmedbio.2012.06.004

23. Hayashi D, Kawakami K, Ito K, et al. Low-energy extracorporeal shock wave therapy enhances skin wound healing in diabetic mice: a critical role of endothelial nitric oxide synthase. Wound Repair Regen Off Publ Wound Heal Soc Eur Tissue Repair Soc. 2012;20 (6):887-895. doi:10.1111/j.1524-475X.2012.00851.x

24. Goertz O, Lauer H, Hirsch T, et al. Extracorporeal shock waves improve angiogenesis after full thickness burn. Burns J Int Soc Burn Inj. 2012;38(7):1010-1018. doi:10.1016/j.burns.2012.02.018

25. Davis TA, Stojadinovic A, Anam K, et al. Extracorporeal shock wave therapy suppresses the early proinflammatory immune response to a severe cutaneous burn injury. Int Wound J. 2009;6(1):11-21. doi:10.1111/j.1742-481X.2008.00540.x

26. Ciampa AR, de Prati AC, Amelio E, et al. Nitric oxide mediates anti-inflammatory action of extracorporeal shock waves. FEBS Lett. 2005;579(30):6839-6845. doi:10.1016/j.febslet.2005.11.023

27. Link KA, Koenig JB, Silveira A, Plattner BL, Lillie BN. Effect of unfocused extracorporeal shock wave therapy on growth factor gene expression in wounds and intact skin of horses. Am J Vet Res. 2013;74(2):324-332. doi:10.2460/ajvr.74.2.324

28. Mariotto S, de Prati AC, Cavalieri E, Amelio E, Marlinghaus E, Suzuki H. Extracorporeal shock wave therapy in inflammatory diseases: molecular mechanism that triggers anti-inflammatory action. Curr Med Chem. 2009;16(19):2366-2372. doi:10.2174/092986709788682119

29. Hausner T, Pajer K, Halat G, et al. Improved rate of peripheral nerve regeneration induced by extracorporeal shock wave treatment in the rat. Exp Neurol. 2012;236(2):363-370. doi:10.1016/j.expneurol.2012.04.019

30. Yamaya S, Ozawa H, Kanno H, et al. Low-energy extracorporeal shock wave therapy promotes vascular endothelial growth factor expression and improves locomotor recovery after spinal cord injury. J Neurosurg. 2014;121(6):1514-1525. doi:10.3171/2014.8.JNS132562

31. Yahata K, Kanno H, Ozawa H, et al. Low-energy extracorporeal shock wave therapy for promotion of vascular endothelial growth factor expression and angiogenesis and improvement of locomotor and sensory functions after spinal cord injury. J Neurosurg Spine. 2016;25(6):745-755. doi:10.3171/2016.4.SPINE15923

32. Kang N, Zhang J, Yu X, Ma Y. Radial extracorporeal shock wave therapy improves cerebral blood flow and neurological function in a rat model of cerebral ischemia. Am J Transl Res. 2017;9(4):2000-2012.

33. Shin D-C, Ha K-Y, Kim Y-H, Kim J-W, Cho Y-K, Kim S-I. Induction of endogenous neural stem cells by extracorporeal shock waves after spinal cord injury. Spine. 2018;43(4):E200-E207. doi:10.1097/ BRS.0000000000002302

34. Lee J-H, Kim S-G. Effects of extracorporeal shock wave therapy on functional recovery and neurotrophin- 3 expression in the spinal cord after crushed sciatic nerve injury in rats. Ultrasound Med Biol. 2015;41(3):790-796. doi:10.1016/j.ultrasmedbio.2014.10.015

35. Zhang J, Kang N, Yu X, Ma Y, Pang X. Radial extracorporeal shock wave therapy enhances the proliferation and differentiation of neural stem cells by notch, PI3K/AKT, and Wnt/ $\beta$-catenin signaling. Sci Rep. 2017;7. doi:10.1038/s41598-017-15662-5

36. Schuh CM, Hausner T, Redl HR. A therapeutic shock propels Schwann cells to proliferate in peripheral nerve injury. Brain Circ. 2016;2(3):138-140. doi:10.4103/2394-8108.192520

37. Wu Y-H, Liang H-W, Chen W-S, Lai J-S, Luh -J-J, Chong F-C. Electrophysiological and functional effects of shock waves on the sciatic nerve of rats. Ultrasound Med Biol. 2008;34(10):1688-1696. doi:10.1016/j.ultrasmedbio.2008.03.005 
38. Lee J-H, Cho S-H. Effect of extracorporeal shock wave therapy on denervation atrophy and function caused by sciatic nerve injury. J Phys Ther Sci. 2013;25(9):1067-1069. doi:10.1589/jpts.25.1067

39. ISMST IS for MST. Shockwave Indications. Available from: https:// www.shockwavetherapy.org/about-eswt/indications/.

40. Lee J-Y, Kim S-N, Lee I-S, Jung H, Lee K-S, Koh S-E. Effects of extracorporeal shock wave therapy on spasticity in patients after brain injury: a meta-analysis. J Phys Ther Sci. 2014;26(10):1641-1647. doi:10.1589/jpts.26.1641

41. Guo P, Gao F, Zhao T, Sun W, Wang B, Li Z. Positive effects of extracorporeal shock wave therapy on spasticity in poststroke patients: a meta-analysis. J Stroke Cerebrovasc Dis Off J Natl Stroke Assoc. 2017;26 (11):2470-2476. doi:10.1016/j.jstrokecerebrovasdis.2017.08.019

42. Xiang J, Wang W, Jiang W, Qian Q. Effects of extracorporeal shock wave therapy on spasticity in post-stroke patients: a systematic review and meta-analysis of randomized controlled trials. J Rehabil Med. 2018. doi:10.2340/16501977-2385

43. Mori L, Marinelli L, Pelosin E, et al. Shock waves in the treatment of muscle hypertonia and dystonia. Biomed Res Int. 2014;2014:1-9. doi: $10.1155 / 2014 / 637450$

44. Dymarek R, Ptaszkowski K, Słupska L, Halski T, Taradaj J, Rosińczuk J. Effects of extracorporeal shock wave on upper and lower limb spasticity in post-stroke patients: a narrative review. Top Stroke Rehabil. 2016;1-11. doi:10.1080/10749357.2016.1141492

45. Amelio E, Manganotti P. Effect of shock wave stimulation on hypertonic plantar flexor muscles in patients with cerebral palsy: a placebo-controlled study. J Rehabil Med. 2010;42(4):339-343. doi:10.2340/16501977-0522

46. Park D-S, Kwon DR, Park G-Y, Lee MY. Therapeutic effect of extracorporeal shock wave therapy according to treatment session on gastrocnemius muscle spasticity in children with spastic cerebral palsy: a pilot study. Ann Rehabil Med. 2015;39(6):914-921. doi:10.5535/arm.2015.39.6.914

47. Picelli A, Marchina EL, Gajofatto F, et al. Sonographic and clinical effects of botulinum toxin Type A combined with extracorporeal shock wave therapy on spastic muscles of children with cerebral palsy. Dev Neurorehabilitation. 2016:1-5. doi:10.3109/17518423.2015.1105320

48. El-Shamy SM, Eid MA, El-Banna MF. Effect of extracorporeal shock wave therapy on gait pattern in hemiplegic cerebral palsy: a randomized controlled trial. Am J Phys Med Rehabil Assoc Acad Physiatr. 2014;93 (12):1065-1072. doi:10.1097/PHM.0000000000000133

49. Vidal X, Morral A, Costa L, Tur M. Radial extracorporeal shock wave therapy (rESWT) in the treatment of spasticity in cerebral palsy: a randomized, placebo-controlled clinical trial. NeuroRehabilitation. 2011;29(4):413-419. doi:10.3233/NRE-2011-0720

50. Gonkova MI, Ilieva EM, Ferriero G, Chavdarov I. Effect of radial shock wave therapy on muscle spasticity in children with cerebral palsy. Int J Rehabil Res Int Z Für Rehabil Rev Int Rech Réadapt. 2013;36(3):284-290. doi:10.1097/MRR.0b013e328360e51d

51. Wang T, Du L, Shan L, et al. A prospective case-control study of radial extracorporeal shock wave therapy for spastic plantar flexor muscles in very young children with cerebral palsy. Medicine (Baltimore). 2016;95 (19):e3649. doi:10.1097/MD.0000000000003649

52. Mori L, Marinelli L, Pelosin E, et al. Radial shock wave therapy: effect on pain and motor performance in a paralympic athlete. Eur J Phys Rehabil Med. 2017;53(2):286-289. doi:10.23736/S19739087.16.03991-5

53. Marinelli LM. Effect of radial shock wave therapy on pain and muscle hypertonia: a double-blind study in patients with multiple sclerosis. Mult Scler Houndmills Basingstoke Engl. 2014;21:5. doi: $10.1177 / 1352458514549566$

54. Wu Y-T, Chang C-N, Chen Y-M, Hu G-C. Comparison of the effect of focused and radial extracorporeal shock waves on spastic equinus in patients with stroke: a randomized controlled trial. Eur J Phys Rehabil Med. 2018;54(4):518-525. doi:10.23736/S1973-9087.17. 04801-8
55. Moher D, Liberati A, Tetzlaff J, Altman DG. Preferred reporting items for systematic reviews and meta-analyses: the PRISMA statement. BMJ. 2009;339:b2535. doi:10.1136/bmj.b2535

56. Moher D, Shamseer L, Clarke M, et al. Preferred reporting items for systematic review and meta-analysis protocols (PRISMA-P) 2015 statement. Syst Rev. 2015;4:1. doi:10.1186/2046-4053-4-1

57. Page MJ, Shamseer L, Tricco AC. Registration of systematic reviews in PROSPERO: 30,000 records and counting. Syst Rev. 2018;7. doi:10.1186/s13643-018-0699-4

58. Booth A, Clarke M, Ghersi D, Moher D, Petticrew M, Stewart L. An international registry of systematic-review protocols. Lancet Lond Engl. 2011;377(9760):108-109. doi:10.1016/S0140-6736(10)60903-8

59. de Morton NA. The PEDro scale is a valid measure of the methodological quality of clinical trials: a demographic study. Aust J Physiother. 2009;55(2):129-133. doi:10.1016/S0004-9514(09)70043-1

60. Burns PB, Rohrich RJ, Chung KC. The levels of evidence and their role in evidence-based medicine. Plast Reconstr Surg. 2011;128 (1):305-310. doi:10.1097/PRS.0b013e318219c171

61. Santamato A, Notarnicola A, Panza F, et al. SBOTE study: extracorporeal shock wave therapy versus electrical stimulation after botulinum toxin type a injection for post-stroke spasticity-a prospective randomized trial. Ultrasound Med Biol. 2013;39(2):283-291. doi:10.1016/j. ultrasmedbio.2012.09.019

62. Yoon SH, Shin MK, Choi EJ, Kang HJ. Effective site for the application of extracorporeal shock-wave therapy on spasticity in chronic stroke: muscle belly or myotendinous junction. Ann Rehabil Med. 2017;41(4):547-555. doi:10.5535/arm.2017.41.4.547

63. Taheri P, Vahdatpour B, Mellat M, Ashtari F, Akbari M. Effect of extracorporeal shock wave therapy on lower limb spasticity in stroke patients. Arch Iran Med. 2017;20(6):338-343. doi:10.172006/AIM.004

64. Lee CH, Lee S-U, Lee SH, Yoo J-I. Ultrasonographic evaluation for the effect of extracorporeal shock wave therapy on gastrocnemius muscle spasticity in patients with chronic stroke. Pm R. 2018. doi:10.1016/j.pmrj.2018.08.379

65. Daliri SS, Forogh B, Emami Razavi SZ, Ahadi T, Madjlesi F, Ansari NN. A single blind, clinical trial to investigate the effects of a single session extracorporeal shock wave therapy on wrist flexor spasticity after stroke. NeuroRehabilitation. 2015;36(1):67-72. doi:10.3233/NRE-141193

66. Li T-Y, Chang C-Y, Chou Y-C, et al. Effect of radial shock wave therapy on spasticity of the upper limb in patients with chronic stroke: a prospective, randomized, single blind, controlled trial. Medicine (Baltimore). 2016;95(18):e3544. doi:10.1097/MD.0000000000003544

67. Radinmehr H, Nakhostin Ansari N, Naghdi S, Olyaei G, Tabatabaei A. Effects of one session radial extracorporeal shockwave therapy on post-stroke plantarflexor spasticity: a single-blind clinical trial. Disabil Rehabil. 2016;1-8. doi:10.3109/09638288.2016.1148785

68. Kim SH, Ha KW, Kim YH, et al. Effect of radial extracorporeal shock wave therapy on hemiplegic shoulder pain syndrome. Ann Rehabil Med. 2016;40(3):509-519. doi:10.5535/arm.2016.40.3.509

69. Dymarek R, Taradaj J, Rosińczuk J. The effect of radial extracorporeal shock wave stimulation on upper limb spasticity in chronic stroke patients: a single-blind, randomized, placebo-controlled study. Ultrasound Med Biol. 2016;42:1862-1875. doi:10.1016/j.ultrasmedbio.2016.03.006

70. Manganotti P, Amelio E. Long-term effect of shock wave therapy on upper limb hypertonia in patients affected by stroke. Stroke. 2005;36 (9):1967-1971. doi:10.1161/01.STR.0000177880.06663.5c

71. Sohn MK, Cho KH, Kim Y-J, Hwang SL. Spasticity and electrophysiologic changes after extracorporeal shock wave therapy on gastrocnemius. Ann Rehabil Med. 2011;35(5):599. doi:10.5535/arm.2011.35.5.599

72. Moon SW, Kim JH, Jung MJ, et al. The effect of extracorporeal shock wave therapy on lower limb spasticity in subacute stroke patients. Ann Rehabil Med. 2013;37(4):461-470. doi:10.5535/arm.2013.37.4.461

73. Santamato A, Micello MF, Panza F, et al. Extracorporeal shock wave therapy for the treatment of poststroke plantar-flexor muscles spasticity: a prospective open-label study. Top Stroke Rehabil. 2014;21 (Suppl 1):S17-S24. doi:10.1310/tsr21S1-S17 
74. Dymarek R, Taradaj J, Rosińczuk J. Extracorporeal shock wave stimulation as alternative treatment modality for wrist and fingers spasticity in poststroke patients: a prospective, open-label, preliminary clinical trial. Evid-Based Complement Altern Med ECAM. 2016;2016:4648101. doi:10.1155/2016/4648101

75. Sawan S, Abd-Allah F, Hegazy MM, Farrag MA, El-Den NHS. Effect of shock wave therapy on ankle plantar flexors spasticity in stroke patients. NeuroRehabilitation. 2017;40(1):115-118. doi:10.3233/NRE-161396

76. Armijo-Olivo S, da Costa BR, Cummings GG, et al. PEDro or cochrane to assess the quality of clinical trials? A meta-epidemiological study. PLoS One. 2015;10:7. doi:10.1371/journal.pone.0132634

77. Bhogal SK, Teasell RW, Foley NC, Speechley MR. The PEDro scale provides a more comprehensive measure of methodological quality than the Jadad scale in stroke rehabilitation literature. J Clin Epidemiol. 2005;58(7):668-673. doi:10.1016/j.jclinepi.2005.01.002
78. Fleuren JFM, Voerman GE, Erren-Wolters CV, et al. Stop using the Ashworth scale for the assessment of spasticity. J Neurol Neurosurg Psychiatry. 2010;81(1):46-52. doi:10.1136/jnnp.2009. 177071

79. Mutlu A, Livanelioglu A, Gunel MK. Reliability of ashworth and modified ashworth scales in children with spastic cerebral palsy. BMC Musculoskelet Disord. 2008;9:44. doi:10.1186/1471-24749-44

80. Meseguer-Henarejos A-B, Sánchez-Meca J, López-Pina J-A, CarlesHernández R. Inter- and intra-rater reliability of the modified Ashworth scale: a systematic review and meta-analysis. Eur J Phys Rehabil Med. 2018;54(4):576-590. doi:10.23736/S1973-9087.17. 04796-7
Clinical Interventions in Aging

\section{Publish your work in this journal}

Clinical Interventions in Aging is an international, peer-reviewed journal focusing on evidence-based reports on the value or lack thereof of treatments intended to prevent or delay the onset of maladaptive correlates of aging in human beings. This journal is indexed on PubMed Central, MedLine, CAS, Scopus and the Elsevier

\section{Dovepress}

Bibliographic databases. The manuscript management system is completely online and includes a very quick and fair peer-review system, which is all easy to use. Visit http://www.dovepress.com/ testimonials.php to read real quotes from published authors. 